\title{
On the Log-Logistic Distribution and Its Generalizations: A Survey
}

\author{
Abdisalam Hassan Muse ${ }^{1}$, Samuel M. Mwalili ${ }^{1,2}$, Oscar Ngesa ${ }^{1,2,3}$ \\ ${ }^{1}$ Mathematics Department, Pan African University, Institute of Basic Sciences, Technology, and Innovation, Kenya \\ ${ }^{2}$ Statistics and Actuarial Sciences Department, Jomo Kenyatta University of Agriculture and Technology, Kenya \\ ${ }^{3}$ Mathematics and Informatics Department, Taita-Taveta University, Kenya \\ Correspondence: Abdisalam Hassan Muse, Mathematics Department (Statistics Option), Pan African University, \\ Institute of Basic Sciences, Technology, and Innovation, (PAUSTI), P.O. Box 6200000200 Nairobi, Kenya.
}

\author{
Received: February 4, 2021 Accepted: April 7, 2021 Online Published: April 20, 2021 \\ doi:10.5539/ijsp.v10n3p93 URL: https://doi.org/10.5539/ijsp.v10n3p93
}

\begin{abstract}
In this paper, we present a review on the log-logistic distribution and some of its recent generalizations. We cite more than twenty distributions obtained by different generating families of univariate continuous distributions or compounding methods on the log-logistic distribution. We reviewed some log-logistic mathematical properties, including the eight different functions used to define lifetime distributions. These results were used to obtain the properties of some log-logistic generalizations from linear representations. A real-life data application is presented to compare some of the surveyed distributions.
\end{abstract}

Keywords: log-logistic distribution, log-logistic generalizations, generalized classes of distributions, construction of new families, censored data, survival analysis

\section{Introduction}

The log-logistic distribution, also known as Fisk distribution in economics, is one of the important continuous probability distributions with a heavy tail defined by one scale (or one rate) and one shape parameters. The log-logistic distribution is a distribution with a non-negative random variable whose logarithm has the very popular logistic distribution. It was initially introduced to model population growth by (Verhulst, 1838). It is often applied to model random lifetimes, and hence has applications in time-to-event analysis. So; if the original data of variable is $x_{1}, x_{2}, x_{3}, \ldots$, etc., then $\log \left(x_{1}\right), \log \left(x_{2}\right), \log \left(x_{3}\right), \ldots$, etc. follow logistic distribution. A logarithmic transformation on the logistic. The LL distribution is similar in shape to the 2-parameter log-normal distribution but it is more suitable for use in the time-to-event data analysis since it has heavier tails than the 2-parameter log-normal. The good thing for log-logistic distribution is that it has greater mathematical tractability when dealing with incomplete (or censored) data and also its cumulative distribution can be written in closed form. Log-logistic distribution is particularly applicable to model heavy tailed data in business, medicine, economics, income, wealth, and social sciences. It can also be found in modeling non-monotone (i.e., unimodal) hazard functions.

The log-logistic distribution has various important properties compared to many other parametric distributions used in the field of survival and reliability analysis: (i) it is cumulative distribution function (cdf) has an explicit closed-from expression, which is very useful for analyzing time-to-event data with incomplete information (e.g. censoring and truncation); (ii) it has a similar shape of pdf and hazard function as the log-normal distribution but has heavier-tails and the tail properties are what the inference is based on; (iii) it has a non-monotonic hazard function: the hazard function is unimodal when shape parameter is greater than 1 and is decreasing monotonically when shape parameter is less than or equal to 1; this is what makes to be different from the Weibull distribution; (iv) it has the potential for analysis of time-to-event data whose rate increases initially and decreases later; (v) it is also used to analyse the skewed data; (vi) the LL distribution can be adopted as the basis of an accelerated failure time (AFT) model by allowing the scale parameter $\alpha$ to differ between groups (Reath et al. 2018), (vii) it has also closed under the proportional odds model; and the last but not the least (viii) The generalization of the LL distribution has an attractive feature of being a member to both AFT and Proportional hazard (PH) models. These important properties are what makes that the log-logistic distribution can be viewed as a simple while useful parametric model which can be widely used in many different disciplines, including demography for modeling population growth (Verhulst, 1838); economics for the distribution of wealth or income inequality (Fisk, 1961); engineering for reliability analysis (Ashkar \& Mahdi, 2003); and hydrology for modelling stream flow rates and precipitation (Rowinski et al. 2002) and many other fields. 
Some other authors who discussed and studied the properties and applications of LL distribution are (Kleiber and Kotz, 2003) studied the application of LL distribution in economics. Collatt (2003) discussed the application of LL distribution in health science for modeling the time following for heart transplantation, Tahir et al (2014) discussed it is useful for modeling censored data usually common in survival and reliability experiments. Other authors who studied the applications of LL distribution are (Prentice, 1976) (Prentice and Kalbfleisch, 1979); (Bennett, 1983); (Singh and George, 1988); (Nandram, 1989); (Diekmann, 1992); (Bacon, 1993); (Little et al. 1994); (BRÜEDERL and Diekmann, 1995); (Gupta et al. 1999) among others. The LL distribution has been widely used in different fields such as actuarial science, economics, survival analysis, reliability analysis, hydrology and engineering. In some cases, the log-logistic distribution is verified to be a good alternative to the log-normal distribution for modeling censored data in survival and reliability analysis due to its mathematical simplicity and it is characterizing increasing hazard rate function. However due to the symmetry of the log-logistic model, it may be poor when the hazard rate is heavily tailed or skewed. Therefore, there is an increasing trend in generalization of the baseline LL distribution by adding an extra shape parameter to the parent (or baseline) distribution or by using other generalization techniques. In the statistical literature, proposing new probability distributions is rich and growing rapidly and various are the papers extending the LL distribution designed to serve as statistical models for a wide range of real lifetime applications with does not follow any of the existing probability distributions.

The remainder of the paper is organized as follows. Section 2 reviews the LL distribution, and the two common parametrization methods for LL distribution and some mathematical properties of the LL distribution are discussed. Section 3 discussed the extensions of the LL distribution. Section 4 methods for generating new families of continuous probability distributions and we cite telegraphically twenty distributions obtained by different generated families and compounding methods on the log-logistic distribution. Section 5 presents the estimation of the parameters. A real-life data application is presented in section 6. Section 7 we discussed the censored data and the G- families of the distributions. concluding remarks and the summary of the work is presented in section 8 . Finally, Section 9 we highlight some future works after the survey.

\section{Log-logistic Distribution}

There are several different parametrizations of the distribution in use. In this study we focused on the two common ones; scale parametrization and rate parametrization. There are several functions related to continuous probability distributions. In this study, we focused on those functions which are related to lifetime distributions as a random variable. The most common ones are; cumulative distribution function (cdf), probability density function (pdf), survival (reliability function), hazard (failure) rate function (HR), cumulative hazard rate (CHR) function, cumulative hazard rate average function (HRA), and the conditional survival function (CSF). The good thing for these functions is that they completely describe the distribution of lifetime, and if you know any of these functions, it is easy to determine the others.

For a random variable $X$ has a log-logistic distribution having shape parameter $\beta>0$, and scale parameter $\alpha>$ 0 , denoted by $\mathrm{X} \sim \operatorname{LL}(\alpha, \beta)$. The cdf, pdf, survivor function, hazard (failure) rate function, cumulative hazard rate function, reversed hazard rate function, the hazard rate average function, and the conditional survival functions are given by respectively:

$$
\begin{gathered}
F(x ; \alpha, \beta)=\frac{1}{1+\left(\frac{x}{\alpha}\right)^{-\beta}}=\frac{\left(\frac{x}{\alpha}\right)^{\beta}}{1+\left(\frac{x}{\alpha}\right)^{\beta}}=\frac{x^{\beta}}{\alpha^{\beta}+x^{\beta}} \\
f(x ; \alpha, \beta)=\frac{\left(\frac{\beta}{\alpha}\right)\left(\frac{x}{\alpha}\right)^{\beta-1}}{\left(1+\left(\frac{x}{\alpha}\right)^{\beta}\right)^{2}} \\
s(x ; \alpha, \beta)=1-F(x ; \alpha, \beta)=\frac{\left(\frac{x}{\alpha}\right)^{-\beta}}{1+\left(\frac{x}{\alpha}\right)^{-\beta}}=\frac{1}{1+\left(\frac{x}{\alpha}\right)^{\beta}} \\
h(x ; \alpha, \beta)=\frac{f(x ; \alpha, \beta)}{S(x ; \alpha, \beta)}=\frac{f(x ; \alpha, \beta)}{1-F(x ; \alpha, \beta)}=\frac{\left(\frac{\beta}{\alpha}\right)\left(\frac{x}{\alpha}\right)^{\beta-1}}{1+\left(\frac{x}{\alpha}\right)^{\beta}},
\end{gathered}
$$




$$
\begin{gathered}
H(x)=-\log R(x)=\int_{0}^{x} h(x) d x, \\
r(x ; \alpha, \beta)=\frac{f(x ; \alpha, \beta)}{F(x ; \alpha, \beta)}==\frac{\left[\left(\frac{\beta}{\alpha}\right)\left(\frac{x}{\alpha}\right)^{\beta-1}\right]}{\left[\frac{\left.\left(\frac{x}{\alpha}\right)^{\beta}\right)^{2}}{\alpha}\right]}=\frac{\left(\frac{\beta}{\alpha}\right)\left(\frac{x}{\alpha}\right)^{-1}}{1+\left(\frac{x}{\alpha}\right)^{\beta}} \\
H R A(x)=\frac{H(x)}{x}=\frac{\int_{0}^{x} h(x) d x .}{x}, x>0, \\
C S F=P(X>x+t \mid X>t)=R(x \mid t)=\frac{R(x+t)}{R(x)}, \quad t>0, \quad x>0 R(.)>0,
\end{gathered}
$$

Where $F(x ; \alpha, \beta)$ is the $\mathrm{CDF}$ of $\mathrm{x}$ analogous to $\mathrm{H}(\mathrm{x})$ in $\mathrm{HRA}(\mathrm{x})$.

\subsection{Alternative Parametrization}

An alternative parametrization is given by applying the rate parameter which is commonly used in some families like the exponential distribution (is the reciprocal of the scale parameter $\alpha$ ) $\rho=\frac{1}{\alpha}$

Therefore, without loss of generality the cumulative density function, probability density function, survivor function and hazard function of the LL distribution are, respectively

$$
\begin{aligned}
& F(x)=\frac{1}{1+(\rho x)^{-\beta}}, \\
& f(x)=\frac{\beta \rho(\rho x)^{\beta-1}}{\left(1+(\rho x)^{\beta}\right)^{2}}, \\
& S(x)=\frac{1}{1+(\rho x)^{\beta}}, \\
& h(x)=\frac{\beta \rho(\rho x)^{\beta-1}}{1+(\rho x)^{\beta}},
\end{aligned}
$$

Where $\rho>0$ and $\beta>0$ are the unknown parameters, $x>0$ is the support of the distribution, and $\beta>0 . \rho$ is the rate parameter and $\beta$ is the shape parameter, that shows as that $\log$-logistic distribution is monotone decreasing when $\beta \leq 1$, and is unimodal when $\beta>1$. If T has a LL distribution, then $\mathrm{Y}=\log \mathrm{T}$ has a logistic distribution.

\subsection{Mean Residual Life Function}

The mean residual life (MRL) function has been widely used in survival and reliability analysis because of its easy interpretability and large area of application. The MRL function computes the expected remaining survival time of a subject given that a component has survived or not failed until time $t$.

Suppose that $F(0)=0$ and $\mu \equiv E(X)=\int_{0}^{\infty} S(x) d x<\infty$. Then the MRL function for continuous X is computed by

$$
m(t \mid \theta)=E(T-t \mid T>t)=\frac{\int_{t}^{\infty}(x-t) f(x \mid \theta) d x}{S(t \mid \theta)}=\frac{\int_{t}^{\infty} S(x \mid \theta) d x}{S(t \mid \theta)}
$$

Where $\theta$ represents the parameter vector of $(\alpha, \beta)$ and $S($.$) is the survival (reliability) function. and m(t \mid \theta) \equiv 0$, whenever $S(t \mid \theta)=0$.

For continuous distributions with finite mean, the survival function is defined through the MRL function:

$$
S(t \mid \theta)=\frac{m(0)}{m(t \mid \theta)} \exp -\left[\int_{0}^{t} \frac{1}{m(x \mid \theta)} d x\right]
$$

Consider the reliability or survival function of the LL distribution with scale and shape parameters. The mean of the LL distribution is only finite when the shape parameter is greater than 1 , thus the mean residual life function is only defined 
when $\beta>1$. The mrl for the LL distribution is easily obtained by

$$
\int_{t}^{\infty} S(x \mid \theta)=\int_{t}^{\infty} \frac{1}{1+(p x)^{k}} d x=\frac{1}{p k} \int_{A(t)}^{1} z^{\frac{1}{k}-1}(1-z)^{-1 / k} d z=\frac{1}{p k}\left[B\left(\frac{1}{k}, 1-\frac{1}{k}\right)-B_{A(t)}\left(\frac{1}{k}, 1-\frac{1}{k}\right)\right],
$$

Where

$$
A(t)=\frac{(p t)^{k}}{\left\{1+(p t)^{k}\right\}}
$$

And

$$
B(p, q)=\int_{0}^{x} y^{p-1}(1-y)^{q-1} d y
$$

Hence the MRL function is given by

$$
m(t \mid \theta)=\frac{1}{p k}\left[B\left(\frac{1}{k}, 1-\frac{1}{k}\right)-B_{A(t)}\left(\frac{1}{k}, 1-\frac{1}{k}\right)\right]\left\{1+(p t)^{k}\right\}, \quad k>1 .
$$

The hazard rate function can also be defined by the MRL function

$$
h(t \mid \theta)=\frac{1+m^{\prime}(t \mid \theta)}{m(t \mid \theta)}
$$

The critical point $t^{*}$ of the hazard rate is given by

$$
t^{*}=\frac{(k-1)^{\frac{1}{k}}}{p}
$$

The hazard rate increases to its maximum at the point $t^{*}$ and then steadily decreases. The mean residual life function has the reverse shape of the hazard rate function. This fact was proved by (Gupta and Akman, 1995).

\subsection{The Quantile Functions}

The quantile functions play a central role in statistical and data analysis. Generally, a probability distribution can be defined either in terms of the distribution function or by the quantile function (Midhu et al. 2013).

The quantile function (inverse CDF) of LL distribution is;

$$
X_{q=} F^{-1}(p ; \alpha, \beta)=\alpha\left(\frac{p}{1-p}\right)^{1 / \beta}, p \in[0,1),
$$

It follows that the

Lower quartile is

Medium is

$$
X_{q_{1}}=3^{-1 / \beta} \alpha
$$

Upper quartile is

$$
\text { Medium }=X_{q_{2}}=\alpha,
$$

$$
X_{q_{3}}=3^{1 / \beta} \alpha
$$

\subsection{Moments and the Moment Generating Function}

Many important properties and features of a probability distribution can be obtained through its moments, such as mean, variance, kurtosis, and skewness. The essential moment functions, such as the moment generating function, $\mathrm{r}^{\text {th }}$ moment, $\mathrm{r}^{\text {th }}$ central moment, are presented.

Theorem 4.3.2: If $T \sim L L(\alpha, \beta)$, then the moment generating function, $1^{\text {st }}$ moment, $2^{\text {nd }}$ moment, and $\mathrm{r}^{\text {th }}$ moments are given, respectively by

$$
M_{X}(t)=\sum_{n=0}^{\infty} \frac{(t \alpha)^{n}}{n !} \cdot \boldsymbol{B}\left(\frac{\beta+n}{\beta}, \frac{\beta-n}{\beta}\right)
$$




$$
\begin{gathered}
M_{X}^{\prime}(t)=\sum_{n=0}^{\infty} \frac{n t^{n-1}}{\alpha^{n} \cdot n !} \cdot \boldsymbol{B}\left(\frac{\beta+n}{\beta}, \frac{\beta-n}{\beta}\right) \\
M_{X}^{\prime \prime}(t)=\sum_{n=0}^{\infty} \frac{n(n-1) t^{n-2} \cdot \alpha^{n}}{n !} \cdot \boldsymbol{B}\left(\frac{\beta+n}{\beta}, \frac{\beta-n}{\beta}\right) \\
M^{r}{ }_{X}(t)=\alpha^{r} \cdot \boldsymbol{B}\left(\frac{\beta+r}{\beta}, \frac{\beta-r}{\beta}\right)
\end{gathered}
$$

Where $B$ is the type-II beta function.

Proof: We have that the Moment Generating Function mgf of $T$ according to (Casella and Berger, 2002) is

$$
M_{X}(t)=\int_{-\infty}^{\infty} e^{\rho t} f(x) d t=\int_{0}^{\infty} e^{t x} f(x) d x=\int_{0}^{\infty} e^{t x} \frac{\frac{\beta}{\alpha}\left(\frac{x}{\alpha}\right)^{\beta-1}}{\left(1+\left(\frac{x}{\alpha}\right)^{\beta}\right)^{2}} d x
$$

By using MacLaurin series the equation becomes:

$$
\begin{aligned}
& M_{X}(t)=\int_{0}^{\infty}\left(1+t x+\frac{(t x)^{2}}{2 !}+\frac{(t x)^{3}}{3 !}+\cdots\right) \frac{\beta}{\alpha}\left(\frac{x}{\alpha}\right)^{\beta}\left(\frac{x}{\alpha}\right)^{-1}\left(1+\left(\frac{x}{\alpha}\right)^{\beta}\right)^{-2} d x \\
& M_{X}(t)=\int_{0}^{\infty} \frac{\beta}{\alpha}\left(\frac{x}{\alpha}\right)^{\beta}\left(\frac{x}{\alpha}\right)^{-1}\left(1+\left(\frac{x}{\alpha}\right)^{\beta}\right)^{-2} d x \\
& +\int_{0}^{\infty} t x \frac{\beta}{\alpha} \frac{(t x)^{2}}{2 !}\left(\frac{x}{\alpha}\right)^{\beta}\left(\frac{t}{\alpha}\right)^{-1}\left(1+\left(\frac{x}{\alpha}\right)^{\beta}\right)^{-2} d x \\
& +\int_{0}^{\infty} \frac{(t x)^{2}}{2 !} \frac{\beta}{\alpha}\left(\frac{x}{\alpha}\right)^{\beta}\left(\frac{x}{\alpha}\right)^{-1}\left(1+\left(\frac{x}{\alpha}\right)^{\beta}\right)^{-2} d x+\int_{0}^{\infty} \frac{(t x)^{3}}{3 !} \frac{\beta}{\alpha}\left(\frac{x}{\alpha}\right)^{\beta}\left(\frac{x}{\alpha}\right)^{-1}\left(1+\left(\frac{x}{\alpha}\right)^{\beta}\right)^{-2} d x+
\end{aligned}
$$

By substituting $y=\left(\frac{x}{\alpha}\right)^{\beta}$, we find $x=y^{\frac{1}{\beta}} \alpha$ with $d x=\frac{\alpha}{\beta} y^{\frac{1}{\beta}-1} d y$. This means that if boundary of $x=0$ then boundary of $y=0$ and for $x=\infty$, then $y=\infty$. Then, the moment generating function it can be written as follows:

$$
M_{X}(t)=\int_{0}^{\infty} \frac{1}{(1+y)^{2}} d y+t \alpha \int_{0}^{\infty} \frac{y^{\frac{1}{\beta}}}{(1+y)^{2}} d y+(t \alpha)^{2} \int_{0}^{\infty} \frac{y^{\frac{2}{\beta}}}{(1+y)^{2}} d y+(t \alpha)^{3} \int_{0}^{\infty} \frac{y^{\frac{3}{\beta}}}{(1+y)^{2}} d y
$$

By applying Beta Function:

$$
M_{X}(t)=\boldsymbol{B}(1,1)+t \alpha \boldsymbol{B}\left(\frac{\beta+1}{\beta}, \frac{\beta-1}{\beta}\right)+\frac{(t \alpha)^{2}}{2 !} \boldsymbol{B}\left(\frac{\beta+1}{\beta}, \frac{\beta-1}{\beta}\right)+\frac{(t \alpha)^{3}}{3 !}\left(\frac{\beta+1}{\beta}, \frac{\beta-1}{\beta}\right)+\cdots
$$

Therefore, the mgt of LL distribution is:

$$
M_{X}(t)=\sum_{n=0}^{\infty} \frac{(t \alpha)^{n}}{n !} \cdot \boldsymbol{B}\left(\frac{\beta+n}{\beta}, \frac{\beta-n}{\beta}\right)
$$

By differentiating the mgt that we have before then the $1^{\text {st }}$ and $2^{\text {nd }}$ moments of LL distribution are retrieved as follows: $1^{\text {st }}$ moment of LL distribution:

$$
\begin{gathered}
M_{X}^{\prime}(t)=\frac{d}{d t} M_{X}(t) \\
M_{X}^{\prime}(t)=\frac{d}{d t} \sum_{n=0}^{\infty} \frac{(t \alpha)^{n}}{n !} \cdot \boldsymbol{B}\left(\frac{\beta+n}{\beta}, \frac{\beta-n}{\beta}\right) \\
M_{X}^{\prime}(t)=\sum_{n=0}^{\infty} \frac{n t^{n-1} \cdot \alpha^{n}}{n !} \cdot \boldsymbol{B}\left(\frac{\beta+n}{\beta}, \frac{\beta-n}{\beta}\right) \\
M_{X}^{\prime}(t=0)=\alpha \cdot \boldsymbol{B}\left(\frac{\beta+1}{\beta}, \frac{\beta-1}{\beta}\right)
\end{gathered}
$$


$2^{\text {nd }}$ moment of LL distribution:

Similarly, the $r^{t^{h}}$ moment in general, is

$$
\begin{aligned}
& M_{X}^{\prime \prime}(t)=\frac{d^{2}}{d t^{2}} M_{X}(t) \\
& M_{X}^{\prime \prime}(t)=\frac{d^{2}}{d t^{2}} \sum_{n=0}^{\infty} \frac{(t \alpha)^{n}}{n !} \cdot \boldsymbol{B}\left(\frac{\beta+n}{\beta}, \frac{\beta-n}{\beta}\right) \\
& M_{X}^{\prime \prime}(t)=\sum_{n=0}^{\infty} \frac{n(n-1) t^{n-2} \cdot \alpha^{n}}{n !} \cdot \boldsymbol{B}\left(\frac{\beta+n}{\beta}, \frac{\beta-n}{\beta}\right) \\
& M_{X}{ }_{X}(t=0)=\alpha^{2} \cdot \boldsymbol{B}\left(\frac{\beta+2}{\beta}, \frac{\beta-2}{\beta}\right)
\end{aligned}
$$

$$
\begin{aligned}
M_{X}^{r}(t) & =\frac{d^{r}}{d t^{r}} M_{X}(t) \\
M_{X}^{r}(t) & =\frac{d^{r}}{d t^{r}} \sum_{n=0}^{\infty} \frac{(t \alpha)^{n}}{n !} \cdot \boldsymbol{B}\left(\frac{\beta+n}{\beta}, \frac{\beta-n}{\beta}\right) \\
M_{X}^{r}(t) & =\sum_{n=0}^{\infty} \frac{n(n-1)(n-2) \ldots(n-(r-1))(n-r) t^{n-r} \alpha^{n}}{n !} \cdot \boldsymbol{B}\left(\frac{\beta+n}{\beta}, \frac{\beta-n}{\beta}\right) \\
M_{X}^{r}(t) & =\alpha^{r} \cdot \boldsymbol{B}\left(\frac{\beta+r}{\beta}, \frac{\beta-r}{\beta}\right)
\end{aligned}
$$

In addition, the mean, variance, skewness and the kurtosis of the log-logistic distribution are given, respectively by The mean of the LL distribution is

$$
\mu=E(T)=\alpha \cdot B\left(\frac{\beta+1}{\beta}, \frac{\beta-1}{\beta}\right)
$$

The Variance of the LL distribution is

$$
\begin{gathered}
\sigma^{2}=V(T)=E\left(T^{2}\right)-(E(T))^{2} \\
=\alpha^{2} \cdot \boldsymbol{B}\left(\frac{\beta+2}{\beta}, \frac{\beta-2}{\beta}\right)-\left(\alpha \cdot \boldsymbol{B}\left(\frac{\beta+1}{\beta}, \frac{\beta-1}{\beta}\right)\right)^{2} \\
\operatorname{Skewness}[X]=\frac{\left[\boldsymbol{B}\left(\frac{\beta+3}{\beta}, \frac{\beta-3}{\beta}\right)-3 \boldsymbol{B}\left(\frac{\beta+2}{\beta}, \frac{\beta-2}{\beta}\right) \boldsymbol{B}\left(\frac{\beta+1}{\beta}, \frac{\beta-1}{\beta}\right)+2\left(\boldsymbol{B}\left(\frac{\beta+1}{\beta}, \frac{\beta-1}{\beta}\right)\right)^{3}\right]}{\left(\boldsymbol{B}\left(\frac{\beta+2}{\beta}, \frac{\beta-2}{\beta}\right)-\left(\boldsymbol{B}\left(\frac{\beta+1}{\beta}, \frac{\beta-1}{\beta}\right)\right)^{2}\right)^{\frac{3}{2}}} \\
\text { Kurtosis }[X]==\frac{\left[\boldsymbol{B}\left(\frac{\beta+4}{\beta}, \frac{\beta-4}{\beta}\right)-4 \boldsymbol{B}\left(\frac{\beta+1}{\beta}, \frac{\beta-1}{\beta}\right) \boldsymbol{B}\left(\frac{\beta+3}{\beta}, \frac{\beta-3}{\beta}\right)-3\left(\boldsymbol{B}\left(\frac{\beta+2}{\beta}, \frac{\beta-2}{\beta}\right)\right)^{2}\right]}{+12 \boldsymbol{B}\left(\frac{\beta+2}{\beta}, \frac{\beta-2}{\beta}\right)\left(\boldsymbol{B}\left(\frac{\beta+1}{\beta}, \frac{\beta-1}{\beta}\right)\right)^{2}-6\left(\boldsymbol{B}\left(\frac{\beta+1}{\beta}, \frac{\beta-1}{\beta}\right)\right)^{\mathbf{4}}} \\
\left(\boldsymbol{B}\left(\frac{\beta+2}{\beta}, \frac{\beta-2}{\beta}\right)-\left(\alpha \cdot \boldsymbol{B}\left(\frac{\beta+1}{\beta}, \frac{\beta-1}{\beta}\right)\right)^{2}\right)^{2}
\end{gathered}
$$

2.5 Characteristic Function

Proposition 4.4.5: The characteristic function of the LL distribution $\varphi_{X}(t)$ is given by

$$
\varphi_{X}(t)=E\left(e^{i t x}\right)=\int_{0}^{\infty} e^{i t x} f(x) d x
$$


As can be seen from the above proposition, the characteristic function of the LL distribution cannot be computed analytically. However, applying the concept of complex analysis; we can express as:

First, since the $e^{i t x}=\cos (t x)+i \sin (t x) \forall t \in \mathbb{R}$;

$$
\varphi_{X}(t)=\int_{0}^{\infty} \cos t x+i \sin t x\left\{\frac{\frac{\beta}{\alpha}\left(\frac{x}{\alpha}\right)^{\beta-1}}{\left(1+\left(\frac{x}{\alpha}\right)^{\beta}\right)^{2}}\right\} d x
$$

Second, evaluating the above equation into two parts to solve it easily, we get:

i)

$$
\int_{0}^{\infty} \cos t x\left\{\frac{\frac{\beta}{\alpha}\left(\frac{x}{\alpha}\right)^{\beta-1}}{\left(1+\left(\frac{x}{\alpha}\right)^{\beta}\right)^{2}}\right\} d x
$$

ii)

$$
\int_{0}^{\infty} i \sin t x\left\{\frac{\frac{\beta}{\alpha}\left(\frac{x}{\alpha}\right)^{\beta-1}}{\left(1+\left(\frac{x}{\alpha}\right)^{\beta}\right)^{2}}\right\} d x
$$

Third, we can transform $\sin (t x)$ and $\cos (t x)$ by applying MacLaurin Series, then we find the results of each part:

i)

$$
\boldsymbol{B}(1,1)-\frac{(t \alpha)^{2}}{2 !} \boldsymbol{B}\left(\frac{\beta+2}{\beta}, \frac{\beta-2}{\beta}\right)+\frac{(t \alpha)^{4}}{4 !} \boldsymbol{B}\left(\frac{\beta+4}{\beta}, \frac{\beta-4}{\beta}\right)+\frac{(t \alpha)^{6}}{6 !} \boldsymbol{B}\left(\frac{\beta+6}{\beta}, \frac{\beta-6}{\beta}\right)+\cdots
$$

ii)

$$
\begin{gathered}
i t \alpha \boldsymbol{B}\left(\frac{\beta+1}{\beta}, \frac{\beta-1}{\beta}\right)-\frac{(i t \alpha)^{3}}{3 !} \boldsymbol{B}\left(\frac{\beta+3}{\beta}, \frac{\beta-3}{\beta}\right)+\frac{(i t \alpha)^{5}}{5 !} \boldsymbol{B}\left(\frac{\beta+5}{\beta}, \frac{\beta-5}{\beta}\right) \\
-\frac{(i t \alpha)^{7}}{7 !} \boldsymbol{B}\left(\frac{\beta+7}{\beta}, \frac{\beta-7}{\beta}\right)+\cdots
\end{gathered}
$$

Hence, the characteristic function is the sum of the two parts:

$$
\begin{aligned}
\varphi_{X}(t) & =\text { part } i+\text { part } i i \\
\varphi_{-} X(t) & =\boldsymbol{B}(1,1)+i t \alpha \boldsymbol{B}\left(\frac{\beta+1}{\beta}, \frac{\beta-1}{\beta}\right)-\frac{(t \alpha)^{2}}{2 !} \boldsymbol{B}\left(\frac{\beta+2}{\beta}, \frac{\beta-2}{\beta}\right)-\frac{(i t \alpha)^{3}}{3 !} \boldsymbol{B}\left(\frac{\beta+3}{\beta}, \frac{\beta-3}{\beta}\right) \\
+ & \frac{(t \alpha)^{4}}{4 !} \boldsymbol{B}\left(\frac{\beta+4}{\beta}, \frac{\beta-4}{\beta}\right)+\frac{(i t \alpha)^{5}}{5 !} \boldsymbol{B}\left(\frac{\beta+5}{\beta}, \frac{\beta-5}{\beta}\right)-\cdots
\end{aligned}
$$

Therefore, the characteristic function of the LL distribution is:

$$
\varphi_{X}(t)=\sum_{n=0}^{\infty} \frac{(i t \alpha)^{n}}{n !} \boldsymbol{B}\left(\frac{\beta+n}{\beta}, \frac{\beta-n}{\beta}\right)
$$

We can also derive the norm characteristic function of the LL distribution:

$$
\left|\varphi_{X}(t)\right|=\boldsymbol{B}(1,1)=1
$$

Since the value of the norm characteristic function of the LL distribution equals 1 . It shows us that the characteristic function of the LL distribution is a finite function.

More details about the log-logistic distribution can be found in (Ekawati et al. 2015)

\section{Extensions of Log-Logistic Distribution}

In recent years, the ability to propose new probability models to deal with reliability and survival analysis has increased. 
Many extensions (or generalizations) of the log-logistic distribution have been proposed in the last two decades. In terms of applications, the log-logistic distribution and its generalizations have become the most popular models for survival and reliability data. Some recent applications have included: modeling for AIDS and Melanoma data (de Santana, Ortega, Cordeiro, \& Silva, 2012); used for minification process (Gui, 2013); modeling breast cancer data (Ramos et al. 2013); (Tahir et al. 2014); modeling on censored survival data (Lemonte, 2014); modeling time up to first calving of cows (Louzada \& Granzotto, 2016); modeling, inference, and use to a polled Tabapua Race Time up to First Calving Data (Granzotto et al. 2017); modeling positive real data in many areas (Lima \& Cordeiro, 2017); analysing a right-censored data (Shakhatreh, 2018); modeling lung cancer data (Alshangiti, et al. 2016); and modeling of breaking stress data (Aldahlan, 2020).

Because of the increasing interest in terms of applications and methodology, we feel it is timely that a survey is provided of the log-logistic distribution and its generalizations. In this study, we provide such a review. We review in the following sections nearly twenty generalizations. For each generalized one, we try to give expressions for the cdf, pdf, reliability (or survival) function, the failure (or hazard) rate function, the reversed hazard function, the quantile function and the cumulative hazard function. Sometimes not all of these functions are given if they are not stated in the original source or if nice closed form expressions are not known.

In the statistics and probability, the literature on statistical theory abounds in surveys of topics of current interest. Ghitany (1998) provided thorough reviews of the recent modifications of the gamma distribution. Pham and Lai (2007) provided through reviews of the recent generalizations of the Weibull distribution. Nadarajah (2013) provided an extensive survey of the exponentiated Weibull distribution. Li and Nadarajah (2020) provided an extensive review of the student's $t$ distribution and its generalizations. Rahman et al. (2020) provided an expository review of the transmuted probability distributions. Tomy et al. 2020) provided an extensive review of the recent generalizations of the exponential distribution. Dey, et al. (2021) provided an extensive review of the transmuted distributions. We contribute to the literature by reviewing the log-logistic distribution and its generalizations. While it is common to come across reviews which deal with generalizations of an existing distributions, our approach is significantly different. Our focus is on the method. To be the best of our knowledge, there is no other work which attempts to bring together at one place nearly twenty generalizations (or extensions) of the LL distribution. In this work, we have reviewed only univariate log-logistic distribution and related distributions. A future work is to review bivariate, multivariate, matrix variate and complex variate log-logistic distribution.

\section{Review of the Methods for Building New Log-Logistic Distributions}

In this section, we present up-to-date review of the methods for building new families of continuous probability distributions. In probability and applied statisticians have shown great interest in building and generating new generalized probability models that extend well-known probability distributions and are more flexible for data modeling in many different disciplines of applications.in recent decades, some different extensions of continuous distributions have received great attention in the recent literature. Gupta and Kundu (2009) discussed six different techniques for the induction of shape/skewness parameter(s) in probability distributions namely: (1) method of proportional hazard model, (2) method of proportional reversed hazard model, (3) method of proportional cumulants model, (4) method of proportional odds model, (5) method of power transformed model, and (6) method of Azzalini's skewed model. On the other hand, Lee et al. (2013), reviewed the different methods of generating new probability distributions and they focused on the two main techniques; adding parameters and combining existing probability distributions. On the other hand, they discussed three methods developed before 1980s, and they are: (1) method of differential equation, (2) method of transformation, and (3) method of quantile. Then they discussed five generating techniques developed since 1980s, those are : (1) method of adding parameters to an existing distribution, (2) composite method, (3) the beta-generated method, (4) the transformed-transformer (T-X) method, and (5) methods of generating skewed distributions. Tahir and Nadarajah (2015) wrote a review of methods for generating probability distributions with more than 300 reference papers, most of these distributions were introduced in the recent years, (Nadarajah et al. 2013); (Tahir and Cordeiro (2015), (Ahmad et al. 2019) and (De Brito et al. 2019) gives an excellent review on the recent developments of univariate continuous distributions.

This study work offers a survey of recently different methods for developing families of probability distributions. The technique of obtaining a new generalized distribution is of different forms; generally speaking, the five methods developed since 1980s, can be named them as a combination methods because of the reason because of the reason that these techniques attempt to add an extra parameters to an existing probability distribution or combining existing distributions into new distributions (Lee et al., 2013). In this work, we will categorize into two headings; (1) generator method or Parameter Induction method, and (2) Compound method. 


\subsection{Generator Method}

There are several different methods described in the literature used to extend well-known probability distributions. Probably, one of the most popular method is to consider distribution generators and is called generator method (also known as parameter induction method). The method deals with an induction a shape parameter(s) to a baseline (or parent) distribution to improve goodness-of-fits and to explore tail properties. In the generator method, we can categorize into; exponentiated-G family, beta-G family, gamma-G family, Marshall-Olkin class, Kumaraswamy class, transmuted family, cubic transmuted family, a general transmuted family, alpha-power transformation, T-X family method, Zubair-G family, and the Cordeiro-Tahir's family.

\subsubsection{The Exponentiated-G family of Distributions}

The exponentiated-G family of distributions can be traced back to Gompertz (1825), (Verhulst 1838;1845;1847) and Lehmann (1953). It is one of the simplest methods for parameter induction techniques. The method adds one shape parameter to an existing distribution. If $G(x)$ is the cumulative distribution function (cdf) of the baseline model, then

$$
F(x)=G(x)^{\gamma}, \gamma>0
$$

$F(x)$ is also the cdf of the new distribution and it is called the exponentiated-G distribution (Exp-G distribution) with exponent parameter $\gamma$.

The distribution $\mathrm{G}$ is the baseline distribution and $\gamma$ is a positive real parameter. The variable $x$ can take any of the form

$$
x=x-\mu \text { or } x=\frac{x-\mu}{\sigma} \text { or } x=k\left(\frac{x-\mu}{\sigma}\right) \text { or } x=k\left(\frac{x-\mu}{\sigma}\right)^{\frac{1}{\sigma}} .
$$

The probability density function (pdf) corresponding to (32) is

$$
f(x)=\gamma(x) G(x)^{\gamma-1}
$$

These family of distributions became famous after the papers by (Mudholkar \& Srivastava, 1993) exponentiated-Weibull distribution, Gupta et al. (1998) named the family to the proportional reversed hazard rate (PHR) model, and (Gupta and Kundu 1999, 2001; 2002) exponentiated -exponential distribution. This family of distributions is considered in many papers in the literature such as (Mudholkar et al. 1995); (Mudholkar \& Hutson, 1996), (Choudhury, 2005), (Singh et al. 2005), (Nadarajah and Kotz 2006), (Barrios \& Dios, 2012), (Shakil \& Ahsanullah, 2012) and (Gholam, 2013), among many others.

Exponentiated log-logistic distribution

This new extended distribution was developed by (Rosaiah et al. 2006), (Aslam \& Jun, 2010), (Rao et al. 2012) and (Chaudhary \& Kumar, 2014). The cdf of Exponentiated log-logistic distribution (ELL) is given as

$$
F_{E L L}(x)=P_{E L L}(\{y \mid y \leq x\})=\left[\frac{\left(\frac{x}{\alpha}\right)^{\beta}}{1+\left(\frac{x}{\alpha}\right)^{\beta}}\right]^{\gamma} \text { for } x \geq 0
$$

where $\alpha, \beta$, and $\gamma>0$ are the unknown parameters of the model.

The ELL is obtained from the CDF of the log-logistic by $F(x)=G(x)^{\gamma}$. The pdf of ELL is as follows:

$$
f_{E L L}(x)=\frac{\beta \gamma\left(\frac{x}{\alpha}\right)^{\beta \gamma-1}}{\alpha\left(1+\left(\frac{x}{\alpha}\right)^{\beta}\right)^{\gamma+1}} \text { for } x \geq 0
$$

The ELL distribution may be taken as a parametric model for survival analysis, if the lifetimes show a large variability. For any lifetime random variable t, the reliability (survival) functions $S(t)$, the hazard rate function $h(t)$, the reversed hazard rate function $r(t)$ and the cumulative hazard rate function $H(t)$, associated with (34) and (35) are

$$
\begin{aligned}
S_{E L L}(t) & =1-F_{E L L}(t) \\
h_{E L L}(t) & =\frac{f_{E L L}(x)}{1-F_{E L L}(t)}
\end{aligned}
$$




$$
\begin{gathered}
r_{E L L}(t)=\frac{f_{E L L}(x)}{F_{E L L}(x)} \\
H_{E L L}(t)=-\log \left[1-F_{E L L}(t)\right]
\end{gathered}
$$

Two-parameter Exponentiated log-logistic distribution:

Chaudhary $(2007,2019)$ studied the exponentiated log-logistic distribution regarding to the standard log-logistic distribution (when $\alpha=1$ ) and called two-parameter exponentiated log-logistic distribution.

If the cdf of the standard LL distribution is

$$
F(x ; \beta)=\frac{x^{\beta}}{1+x^{\beta}}, x<0, \quad \beta>0
$$

Then the cdf of the two-parameter exponentiated LL distribution is given by

$$
F(x ; \beta)=\left\{\frac{x^{\beta}}{1+x^{\beta}}\right\}^{\gamma}, x<0, \quad \beta>0
$$

the pdf corresponding to (41) is given by

$$
f(x)=\frac{\beta \gamma x^{\beta \gamma}}{x\left[1+x^{\beta}\right]^{\gamma+1}} \text { for } x>0
$$

The survivor function, hazard rate function and the quantile function are given by respectively

$$
\begin{gathered}
h(x)=\frac{\beta \gamma x^{\beta \gamma}}{\left[1-\left\{\frac{x^{\beta}}{1+x^{\beta}}\right\}^{\gamma}\right]} \text { for } x>0 \\
S(x)=1-\left\{\frac{x^{\beta}}{1+x^{\beta}}\right\}^{\gamma} \text { for } x>0 \\
x_{q}=\left(q^{-\frac{1}{\gamma}}-1\right)^{-\frac{1}{\beta}} \quad \text { for } 0<q>1 .
\end{gathered}
$$

\subsubsection{The Beta-G Family of Distributions}

Beta distribution is a continuous probability distribution with two positive shape parameters, $a$ and $b$. It is the natural extension of the uniform distribution and the prior of the binomial distribution. It can rescale and shift to create a new probability distribution with a wide range of shapes and apply for a different application.

Eugene et al. (2002) and Jones (2004)were introduced the Beta-G family of distributions based on the parameter induction technique. The generator method that Eugene et al. (2002) proposed is as follows. For any continuous baseline cumulative distribution function cdf

$$
G(x)=G(x, \theta)
$$

where $\theta$ is the parameter vector, the cdf of the beta-G, $F(x)$ say, is given by

$$
F(x)=I_{G(x)}(a, b)=\frac{1}{B(a, b)} \int_{0}^{G(x)} x^{a-1}(1-x)^{b-1} d x,
$$

Where $a, b>0$ are additional shape parameters to those in $\theta$ that aim to provide greater flexibility of its tails and to introduce skewness. $x \in(0,1), \boldsymbol{B}(a, b)=\int_{0}^{\infty} x^{a-1}(1-x)^{b-1} d x$ represents the beta function and $I_{G(x)}(a, b)=$ $\frac{1}{\boldsymbol{B}(a, b)} \int_{0}^{x} x^{a-1}(1-x)^{b-1} d x$ represents the incomplete beta function ratio, $\boldsymbol{B}(a, b)=\int_{0}^{1} x^{a-1}(1-x)^{b-1} d x=$ $\frac{\Gamma(a)+\Gamma(b)}{\Gamma(a+b)}$ is the beta function, $\boldsymbol{\Gamma}($.$) is the gamma function, and \boldsymbol{B}(a, b)=\int_{0}^{x} x^{a-1}(1-x)^{b-1} d x$ is the incomplete beta function.

The pdf of the beta-G family of distribution takes the form

$$
f(x)=\frac{1}{B(a, b)} g(x) G(x)^{a-1}[1-G(x)]^{b-1}
$$


The beta-G family is also called the beta logit family. These family of distributions become much more popular after Eugene et al. (2002). This class is studied and discussed the estimation methods and the characterization by maximum entropy by (Zografos \& Balakrishnan, 2009) and the moments from beta-G family are studied by (Cordeiro \& Nadarajah, 2011)

Beta log-logistic distribution

Lemonte, (2014) proposed and studied the beta log-logistic distribution. The new distribution is quite flexible to model and analyze positive real data. The cdf of the beta log-logistic (BLL) distribution is given by

$$
F_{B L L}(t)=I_{\frac{x^{\beta}}{\alpha^{\beta}+x^{\beta}}}(a, b)
$$

The pdf corresponding to (48) is

$$
f_{B L L}(x)=\frac{\left(\frac{\beta}{\alpha}\right)}{B(a, b)} \frac{\left(\frac{x}{\alpha}\right)^{a \beta-1}}{\left[1+\left(\frac{x}{\alpha}\right)^{\beta}\right]^{a+b}}, \quad x>0 .
$$

For any lifetime random variable t, the reliability (survival) functions $S(t)$, the hazard rate function $h(t)$, the reversed hazard rate function $r(t)$ and the cumulative hazard rate function $H(t)$, associated with (48) and (49) are

$$
\begin{gathered}
S(t)=1-I_{G(x)}(a, b)=1-I_{\frac{x^{\beta}}{\alpha^{\beta}+x^{\beta}}}(a, b) \\
h(t)=\frac{f_{B L L}(x)}{1-F_{B L L}(t)} \\
r(t)=\frac{f_{B L L}(x)}{F_{B L L}(x)} \\
H(t)=-\log \left[1-F_{B L L}(t)\right]
\end{gathered}
$$

This extension distribution can be used in many fields, like economics, reliability analysis in engineering, survival analysis, hydrology, and other disciplines as the LL distribution.

\subsubsection{The Gamma-G Family of Distributions}

Zografos and Balakrishnan (2009)proposed a simple generator approach (or parameter induction technique). If $G(x, \theta), g(x, \theta)$, and $S(x, \theta)$ are the cdf, pdf and survival function of the baseline distribution respectively. Then the cdf of the gamma-G family is given by

$$
F(x, \theta)=\frac{1}{\Gamma(\delta)} \int_{0}^{-\log s(x, \theta)} t^{\delta-1} e^{-t} d t, \quad \delta>0
$$

And the pdf of the gamma-G family is given by

$$
f(x, \theta)=\frac{1}{\Gamma(\delta)}[-\log S(x, \theta)]^{\delta-1} g(x, \theta) .
$$

The corresponding hazard (or failure) rate function is given by

$$
h(x, \theta)=\frac{1}{\Gamma(\delta,-\log S(x, \theta))}[-\log S(x, \theta)]^{\delta-1} g(x, \theta) .
$$

Another gamma-G family was proposed by (Ristic and Balakrishnan, 2012) which is slightly different from the above generator. The cdf and pdf of their generator was defined by

$$
F(x, \theta)=1-\frac{1}{\Gamma(\delta)} \int_{0}^{-\log S(x, \theta)} t^{\delta-1} e^{-t} d t, \quad \delta>0
$$

And the pdf of the gamma-G family is given by

$$
f(x, \theta)=\frac{1}{\Gamma(\delta)}[-\log F(x, \theta)]^{\delta-1} g(x, \theta) .
$$

The corresponding hazard function is given by 


$$
h(x, \theta)=\frac{1}{\Gamma(\delta,-\log F(x, \theta))}[-\log F(x, \theta)]^{\delta-1} g(x, \theta) .
$$

Nadarajah et al. (2015b) studied the mathematical and statistical properties of the Zografos-Balakrishnan-G family of distributions.

\section{Zografos-Balakrishnan log-logistic distribution}

Ramos et al. (2013) proposed a gamma log-logistic distribution by using the Zografos-Balakrishnan -G technique and they called the Zografos-Balakrishnan Log-logistic distribution (ZB-G). The ZB-G family is a LL distribution plus an extra shape parameter $\delta>0$. The pdf and cdf of the Zografos-Balakrishnan Log-logistic distribution is given by using the equations of (1) and (2):

$$
f(x, \theta)=\frac{\beta}{\alpha^{\beta} \Gamma(\delta)} x^{\beta-1}\left[1+\left(\frac{x}{\alpha}\right)^{\beta}\right]^{-2}\left\{\log \left[1+\left(\frac{x}{\alpha}\right)^{\beta}\right]\right\}^{\delta-1} .
$$

And the corresponding cdf is given by

$$
F(x, \theta)=\frac{1}{\Gamma(\delta)} \int_{0}^{\log \left[1+\left(\frac{x}{\alpha}\right)^{\beta}\right]} t^{\delta-1} e^{-t} d t,
$$

The method for Ristic and Balakrishnan (2012) is an alternative method that can be used to extend the LL distribution and it can be an open research question.

\subsubsection{The Marshall-Olkin Family of Distributions}

Marshall and Olkin, (1997) introduced a simple generator approach of adding a single parameter to a family of well-known distributions and several authors applied their technique to generalize the well-known probability distributions in the last two decades.

If $G(x, \theta)$ and $S(x, \theta)$ are the cdf and survival function of the baseline distribution depending on the vector parameter , and $\gamma>0$ is an additional parameter known as tilt parameter. Then the survival function of Marshall and Olkin (MO) family is given by

$$
S_{M O}(x ; \gamma, \theta)=\frac{\gamma \cdot S(x, \theta)}{1-\bar{\gamma} S(x, \theta)}, \quad \theta, \gamma>0, x \in \mathbb{R} .
$$

Where $\bar{\gamma}=1-\gamma$. Note that, if $\gamma=1$, then $S_{M O}(x, \gamma, \theta)=S(x, \theta)$ which means that we obtain the baseline distribution. Applying (62), the generalized versions of the well-known distribution have been proposed.

The pdf corresponding to (62) is given by

$$
f(x ; \gamma, \theta)=\frac{\gamma g(x ; \theta)}{\{1-\bar{\gamma} S(x, \theta)\}^{2}}
$$

And the hazard rate function $\mathrm{h}(\mathrm{t})$ is given by

$$
h(x ; \gamma, \theta)=\frac{1}{1-\bar{\gamma} S(x, \theta)} h(x ; v=\theta), \quad x \in \mathbb{R}
$$

Marshall-Olkin log-logistic distribution

Gui (2013) introduced the Marshall-Olkin Log-logistic distribution (MOLL) and studied the mathematical and statistical properties of the proposed model and used it to models of time series. The survival function, pdf, and the hazard rate function of the proposed distribution are given by:

$$
\begin{gathered}
S_{M O L L}(x ; \alpha, \beta, \gamma)=\frac{\gamma \cdot \alpha^{\beta}}{x^{\beta}+\gamma \cdot \alpha^{\beta}}, \quad \alpha, \beta, \gamma, x>0, \\
f_{M O L L}(x ; \alpha, \beta, \gamma)=\frac{\alpha^{\beta} \beta \gamma x^{\beta-1}}{\left(x^{\beta}+\gamma \cdot \alpha^{\beta}\right)^{2}}, \quad \alpha, \beta, \gamma, x>0, \\
h_{M O L L}(x ; \alpha, \beta, \gamma)=\frac{\beta x^{\beta-1}}{x^{\beta}+\gamma \cdot \alpha^{\beta}}, \quad \alpha, \beta, \gamma, x>0,
\end{gathered}
$$

Other authors who studied the further results involving reliability analysis, the estimation of the parameters and the uses of the Marshall-Olkin log-logistic distribution are (Alshangiti et al. 2016), and (Shakhatreh, 2018), (Nasiru et al. 
2019)

\subsubsection{The Alpha Power Transformation}

Mahdavi and Kundu (2017) proposed a new generator technique that many authors applied to introduce for new statistical distributions to increase flexibility of the given family. The technique adds a new parameter to the baseline distribution. The cdf of Alpha power transformation (AP) is defined as

$$
F_{A P T}(x ; \theta, \gamma)=\frac{\gamma^{G(x ; \theta)}-1}{\gamma-1} \theta, \gamma>0, \gamma \neq 1, x \in \mathbb{R} .
$$

where $G(x ; \theta)$ is the cdf of the baseline distribution and $\theta$ is the vector parameter.

The pdf corresponding to (68) is given as

$$
F_{A P T}(x ; \theta, \gamma)=\frac{\log (\gamma) \gamma^{G(x ; \theta)}}{\gamma-1} g(x ; \theta) ; \quad \theta, \gamma>0, \gamma \neq 1, x \in \mathbb{R} .
$$

Two-parameter Alpha Power Transformed Log-logistic distribution

Several researchers have applied the alpha power technique to extend the log-logistic distribution.

Malik and Ahmad (2020) proposed the two-parameter alpha power log-logistic distribution (APLL) of two unknown parameters $\theta$ scale parameters and $\gamma$ shape parameter. Note that, Malik and Ahmad (2020) extended the standard $\log$-logistic distribution (where the shape parameter equals 1 ).

If the cdf of the standard LL distribution takes the form:

$$
F(x ; \theta)=\frac{x^{\theta}}{1+x^{\theta}},
$$

Then the cdf of the APLL is given by

$$
F_{A P L L}(x ; \theta, \gamma)=\frac{\gamma\left(\frac{x^{\theta}}{1+x^{\theta}}\right)-1}{\gamma-1} \quad \theta, \gamma>0, \gamma \neq 1, x \in \mathbb{R} .
$$

The pdf corresponding to (71) is given by

$$
f_{A P L L}(x ; \theta, \gamma)=\frac{\log (\gamma)}{\gamma-1} \frac{\theta x^{\theta-1}}{\left(1+x^{\theta}\right)^{2}} \gamma^{\left(\frac{x^{\theta}}{1+x^{\theta}}\right)} ; \quad \theta, \gamma>0, \gamma \neq 1, x \in \mathbb{R} .
$$

The survival function of the APLL is defined as

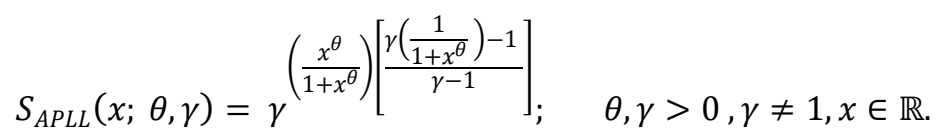

The hazard rate function of the APLL is given by

$$
h_{A P L L}(x ; \theta, \gamma)=\log (\gamma) \frac{\theta x^{\theta-1}}{\left(1+x^{\theta}\right)^{2}} \frac{1}{\gamma\left(\frac{1}{1+x^{\theta}}\right)-1} ; \quad \theta, \gamma>0, \gamma \neq 1, x \in \mathbb{R} .
$$

The reverse hazard rate function of the APLL is given by

$$
r_{A P L L}(x ; \theta, \gamma)=\log (\gamma) \frac{\theta x^{\theta-1}}{\left(1+x^{\theta}\right)^{2}} \frac{\gamma\left(\frac{1}{1+x^{\theta}}\right)}{\gamma\left(\frac{1}{1+x^{\theta}}\right)-1} ; \quad \theta, \gamma>0, \gamma \neq 1, x \in \mathbb{R} .
$$

The Alpha Power Transformed Log-logistic distribution

Aldahlan (2020) proposed an Alpha Power Transformed Log-logistic distribution (APTLL) and studied the mathematical and statistical properties of the new distribution. Aldahlan (2020) extended the two-parameter $\log$-logistic distribution. If the cdf of the two-parameter baseline log-logistic distribution takes the form:

$$
F(x ; \alpha, \beta)=\frac{1}{1+\left(\frac{x}{\alpha}\right)^{-\beta}},
$$


Then the cdf of the APTLL is given by

$$
F_{A P T L L}(x ; \alpha, \beta, \gamma)=\frac{\gamma\left(\frac{1}{1+\left(\frac{x}{\alpha}\right)^{-\beta}}\right)-1}{\gamma-1} \quad \alpha, \beta, \gamma>0, \gamma \neq 1, x>0 .
$$

The pdf corresponding to (77) is given by

$$
f_{A P T L L}(x ; \alpha, \beta, \gamma)=\frac{\log (\gamma)}{\alpha^{\beta}(\gamma-1)} \frac{\beta x^{\beta-1}}{\left(1+\left(\frac{x}{\alpha}\right)^{\beta}\right)^{2}} \gamma^{\left(\frac{1}{1+\left(\frac{x}{\alpha}\right)^{-\beta}}\right)} ; \quad \alpha, \beta, \gamma>0, \gamma \neq 1, x>0 .
$$

The survival function of the APTLL is defined as

$$
S_{A P T L L}(x ; \alpha, \beta, \gamma)=\frac{\gamma-\gamma^{\left(\frac{1}{1+\left(\frac{x}{\alpha}\right)^{-\beta}}\right)}}{\gamma-1} ; \quad \alpha, \beta, \gamma>0, \gamma \neq 1, x>0 .
$$

The hazard rate function of the APTLL is given by

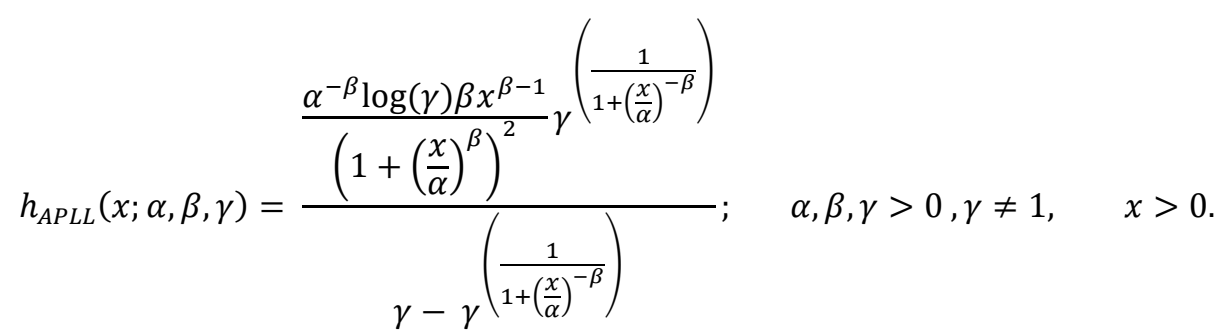

The reverse hazard rate function of the APTLL is given by

$$
r_{A P T L L}(x ; \alpha, \beta, \gamma)=\frac{\frac{\alpha^{-\beta} \log (\gamma) \beta x^{\beta-1}}{\left(1+\left(\frac{x}{\alpha}\right)^{\beta}\right)^{2}} \gamma\left(\frac{1}{1+\left(\frac{x}{\alpha}\right)^{-\beta}}\right)}{\gamma\left(\frac{1}{1+\left(\frac{x}{\alpha}\right)^{-\beta}}\right)-1} ; \quad \alpha, \beta, \gamma>0, \gamma \neq 1, x>0 .
$$

The cumulative hazard rate function of the APTLL is given by

\subsubsection{The Kumaraswamy-G Family of Distributions}

$$
H_{A P T L L}(x ; \alpha, \beta, \gamma)=-\log \left[\frac{\gamma-\gamma^{\left(\frac{1}{1+\left(\frac{x}{\alpha}\right)^{-\beta}}\right)}}{\gamma-1}\right] ; \quad \alpha, \beta, \gamma>0, \gamma \neq 1, x>0 .
$$

Kumaraswamy Distribution

Kumaraswamy (1980) introduced a new two-parameter continuous distribution on $(0,1)$ named Kumaraswamy distribution. Kumaraswamy distribution has the cdf and pdf of the form

$$
G(x)=1-\left(1-x^{a}\right)^{b},
$$

where $x \in(0,1)$ and $a$ and $b$ are both shape parameters.

$$
g(x)=a b x^{a-1}\left(1-x^{a}\right)^{b-1}
$$


The Kumaraswamy distribution has the same basic shape properties to the beta distribution; where (1) $a>1$ and $b>$ 1 unimodal; (2) $a<1$ and $b<1$ bathtub; (3) $a \leq 1$ and $b>1$ decreasing; (4) $a>1$ and $b \leq 1$ increasing, and (4) $a=b=1$ constant.

Kumaraswamy-G family of distributions

Jones (2009) and Cordeiro and de Castro (2011) introduced the Kumaraswamy-G family of distributions by extending the beta-G family of distributions by applying Kumaraswamy distribution as a generator instead of the beta generator. That is; the cdf of the Kumaraswamy-G of distributions is derived by replacing the cdf in (46) by the Kumaraswamy distribution as the following:

The cdf of the Kumaraswamy-G family of distributions

The pdf corresponding to (85) is given by

$$
F_{K U-G}(x)=1-\left[1-G(x)^{a}\right]^{b},
$$

$$
f_{K U-G}(x)=a b g(x) G(x)^{a-1}\left[1-G(x)^{a}\right]^{b-1},
$$

Where $x>0, a, b>0$ are the two extra shape parameters in addition to those in the baseline model whose role are to govern tail weights and skewness. The pdf of the Kumaraswamy-G family has many similar properties to the beta-G family, but has some advantages in terms of mathematical tractability, since it doesn't involve any specific function such as the beta function.

Kumaraswamy log-logistic distribution

de Santana et al. (2012) and Muthulakshmi, (2013) proposed and studied the mathematical and statistical properties of the Kumaraswamy log-logistic distribution. de Santana et al. (2012) proposed a new distribution that contain several essential distributions as sub-models to the extended distribution.

The cdf and pdf of the Kumaraswamy Log-logistic distribution (KULL) are defined as

$$
\begin{gathered}
F_{K U L L}(x)=\left[\left(1-\frac{1}{1+\left(\frac{x}{\alpha}\right)^{\beta}}\right)^{a}\right]^{b}, \\
f_{K U L L}(x)=\frac{a b \beta}{\alpha^{a \beta}} x^{a \beta-1}\left[1+\left(\frac{x}{\alpha}\right)^{\beta}\right]^{-(a+1)}\left\{1-\left[1-\frac{1}{1+\left(\frac{x}{\alpha}\right)^{\beta}}\right]^{a}, \quad x>0\right.
\end{gathered}
$$

The survival function corresponding to (87) is

$$
S_{K U L L}(x)=1-F_{K U L L}(x)=\left[1-\left(1-\frac{1}{1+\left(\frac{x}{\alpha}\right)^{\beta}}\right)^{a}\right]^{b},
$$

The hazard function corresponding to $(87)$ is

$$
h_{K U L L}(x)=\frac{a b \beta}{\alpha^{a \beta}} x^{a \beta-1}\left[1+\left(\frac{x}{\alpha}\right)^{\beta}\right]^{-(a+1)}\left\{1-\left[1-\frac{1}{1+\left(\frac{x}{\alpha}\right)^{\beta}}\right]^{a}, \quad x>0\right.
$$

where $\alpha$ is the scale parameter, and the shape parameters $a, b$, and $\beta$ govern the skewness of (87).

\subsubsection{The McDonald-G family of Distributions}

\section{McDonald Distribution}

McDonald, (2008) introduced a new distribution called McDonald distribution with cdf of the form

$$
F(x ; a, b, c)=I_{x} c\left(a c^{-1}, b\right)=\frac{B_{x} c\left(a c^{-1}, b\right)}{B\left(a c^{-1}, b\right)}=\frac{1}{B\left(a c^{-1}, b\right)} \int_{0}^{x^{c}} x^{a c^{-1}-1}(1-x)^{b-1} d x
$$


where $a, b, c>0$ are the three shape parameters. Some of the special cases of the Mc distribution includes the beta type 1 distribution $(c=1)$ and the Kumaraswamy distribution $(a=1)$.

The pdf corresponding to $(91)$ is

$$
f(x ; a, b, c)=\frac{c}{B\left(a c^{-1}, b\right)} x^{a-1}\left(1-x^{c}\right)^{b-1}, 0<x<1
$$

McDonald-G family of distributions

Alexander et al. (2012) introduced the McDonald-G family of distributions by replacing the upper limit $x^{c}$ of the integral in equation (91) with $G\left(x^{c}\right)$.

For any baseline cumulative distribution function (cdf) $G(x)$, the resulting $\operatorname{cdf} F(x)$ of the Mc-generalized family of distribution Mc-G is

$$
F_{M c G}(x, \theta)=I_{G(x)^{c}}\left(a c^{-1}, b\right)=\frac{1}{B\left(a c^{-1}, b\right)} \int_{0}^{G(x)^{c}} x^{a c^{-1}-1}(1-x)^{b-1} d x
$$

where the $I_{G(x)^{c}}(a, b)$ is the incomplete beta function ratio and $I_{G(x)^{c}}(a, b)=\frac{B_{G(x)^{c}\left(a c^{-1}, b\right)}}{B\left(a c^{-1}, b\right)}$

The pdf corresponding to $(93)$ is

$$
f_{M c G}(x, \theta)=\frac{c}{B\left(a c^{-1}, b\right)} g(x)\{G(x)\}^{a-1}\left\{1-G(x)^{c}\right\}^{b-1}
$$

Lemonte and Cordeiro (2013) stated that this method of parameter induction facilitates the computation of several statistical and mathematical properties of the $\mathrm{G}$ family of probability distributions.

McDonald Log-logistic distribution

Tahir et al. (2014) introduced and studied the McDonald log-logistic distribution and they considered three of the above extended models of log-logistic distribution, (1) beta log-logistic distribution; (2) Kumaraswamy log-logistic distribution; and (3) gamma log-logistic or Zografos-Balakrishnan log-logistic distribution.

Using the equations of the cdf and pdf of the McDonald-G family, they obtained the CDF and pdf of the McDonald log-logistic distribution.

The cdf of the McDonald is given by

$$
F_{M c L L}(x, \theta)=I_{w}\left(a c^{-1}, b\right)=\frac{1}{B(a, b)} \int_{0}^{w^{c}} w^{a c^{-1}-1}(1-w)^{b-1} d w
$$

Where $w=C D F$ of LL distribution $=\left[1+\left(\frac{x}{\alpha}\right)^{-\beta}\right]^{-1}$ and $a, b, c, \alpha, \beta>0, \quad$ where $a, b, c$, and $\beta$ are shape parameters while $\alpha$ is a scale parameter.

The corresponding pdf of the (95) is given by

$$
f_{M c L L}(x, \theta)=\frac{c}{B\left(a c^{-1}, b\right)}\left(\frac{\beta}{\alpha}\right)\left(\frac{x}{\alpha}\right)^{a \beta-1}\left[1+\left(\frac{x}{\alpha}\right)^{\beta}\right]^{-(a+1)}\left[1-\left\{1-\left[1+\left(\frac{x}{\alpha}\right)^{\beta}\right]^{-1}\right\}^{c}\right]^{b-1},
$$

For a lifetime random variable $t$, the survivor function, the hazard (failure) rate function, the reversed hazard rate function, the cumulative hazard rate function and the quantile function of the McDLL distribution are given by

$$
\begin{aligned}
& S(t, \theta)=1-F(t, \theta)=1-I_{w}\left(a c^{-1}, b\right) \\
& h(t, \theta)=\frac{c\left(\frac{\beta}{\alpha}\right)\left(\frac{t}{\alpha}\right)^{a \beta-1}\left[1-\left\{1-\left[1+\left(\frac{t}{\alpha}\right)^{\beta}\right]^{-1}\right\}^{c}\right]^{b-1}}{B\left(a c^{-1}, b\right)\left[1+\left(\frac{t}{\alpha}\right)^{\beta}\right]^{(a+1)}\left[I_{w}\left(a c^{-1}, b\right)\right]}
\end{aligned}
$$




$$
\begin{gathered}
r(t, \theta)=\frac{c\left(\frac{\beta}{\alpha}\right)\left(\frac{t}{\alpha}\right)^{a \beta-1}\left[1-\left\{1-\left[1+\left(\frac{t}{\alpha}\right)^{\beta}\right]^{-1}\right\}^{c}\right]^{b-1}}{B\left(a c^{-1}, b\right)\left[1+\left(\frac{t}{\alpha}\right)^{\beta}\right]^{(a+1)}\left[1-I_{w}\left(a c^{-1}, b\right)\right]} \\
H(t, \theta)=\int_{0}^{t} h(t, \theta) d t=-\log S(t, \theta)=-\log \left[1-I_{w}\left(a c^{-1}, b\right)\right]
\end{gathered}
$$

And

$$
X_{q}=\left\{\left[\left(I_{q}^{-1}\left(a c^{-1}, b\right)\right)^{-\frac{1}{c}}-1\right] \alpha\right\}^{-\frac{1}{\beta}}
$$

\subsubsection{The Transmuted Family of Distributions}

Shaw and Buckley (2009) introduced a new method of parameter induction (generator technique) and several researchers applied their method to extend and generalize the well-known probability distributions in the last decade. They named the family as quadratic transmuted family of distributions and they used it to financial mathematics and other applied fields.

For any baseline distribution with $\operatorname{cdf} G(x ; \theta)$ depending on the vector parameter $\theta$, then the CDF of the transmuted family is given by

$$
F(x ; \gamma, \theta)=(1+\gamma) G(x ; \theta)-\gamma G(x ; \theta)^{2},
$$

where $\theta>0,|\gamma| \geq 1, x \in \mathbb{R} . \quad \theta$ is the vector parameter, $\gamma$ is the extra shape parameter. When $\gamma=0$, we obtain the baseline distribution, i.e., $F(x ; \gamma, \theta)=G(x ; \theta)$.

The pdf corresponding to (102) is given by using differentiation

$$
f(x ; \gamma, \theta)=g(x ; \gamma, \theta)[1+\gamma-2 \gamma G(x ; \theta)],
$$

Aryal and Tsokos $(2009,2011)$ first highlight the method in (114) and proposed a couple of transmuted probability distributions that would offer more distributional flexibility in reliability and environmental analysis. For detail about this family we refer to (Bourguignon et al. 2016)and (Alizadeh et al. 2017) who studied the general properties of this family, while (Tahir and Cordeiro, 2015) have introduced a list of quadratic transmuted family of distributions. Rahman et al. (2020) have provided an up-to-date list of popular transmuted -G classes of distributions.

Transmuted Log-logistic distribution

Aryal (2013) proposed and studied some of the statistical and mathematical properties of the transmuted log-logistic distribution. using the equations (102) and (103); they derived the cdf and pdf of the transmuted LL distribution as follows:

$$
F_{T L L}(x ; \gamma, \theta)=\frac{(1+\gamma) \alpha^{\beta} x^{\beta}+x^{2 \beta}}{\left(\alpha^{\beta}+x^{\beta}\right)^{2}}, x>0
$$

The pdf of the transmuted log-logistic distribution is given by

$$
f_{T L L}(x ; \gamma, \theta)=\frac{\beta \alpha^{\beta} x^{\beta-1}\left[(1+\gamma)\left(\alpha^{\beta}+x^{\beta}\right)+2 \gamma x^{\beta}\right]}{\left(\alpha^{\beta}+x^{\beta}\right)^{3}}, x>0
$$

More details about the transmuted LL distribution and its applications to real data sets can be found in (Granzotto and Louzada, 2015); (Louzada and Granzotto, 2016); and (Adeyinka, 2019).

\subsubsection{The Cubic Transmuted Family of Distributions}

Granzotto et al. (2017) introduced a new parameter induction technique of generating probability distributions called Cubic Transmutation technique. The reason behind developing of cubic transmuted family was that the quadratic transmuted distribution captures the complexity of unimodal data but the real-life data become more complex to use them, and sometimes cannot be fitted by applying the quadratic transmuted family.

For any baseline distribution with $\operatorname{cdf} G(x ; \theta)$ depending on the vector parameter $\theta$, then the CDF of the cubic transmuted family is given by

$$
F(x ; \gamma, \theta)=\gamma_{1} G(x ; \theta)+\left(\gamma_{2}-\gamma_{1}\right) G(x ; \theta)^{2}+\left(1-\gamma_{2}\right) G(x ; \theta)^{3}, \quad x \in \mathbb{R},
$$

where $\gamma_{1} \in[0,1]$, and $\gamma_{2} \in[-1,1]$. 
The pdf corresponding to (106) is given by

$$
f(x ; \gamma, \theta)=g(x ; \theta)\left[\gamma_{1}+2\left(\gamma_{2}-\gamma_{1}\right) G(x ; \theta)+3\left(1-\gamma_{2}\right) G(x ; \theta)^{2}\right], \quad x \in \mathbb{R},
$$

Rahman et al. $(2019,2020)$ proposed two new cubic transmuted families of distributions. On the other hand, Aslam et al. (2018)introduced another cubic transmuted-G family of distributions using the T-X idea of (Alzaatreh et al, 2013).

Rahman et al. (2020) have provided an up-to-date list of popular cubic transmuted -G classes of distributions. In general, the cubic transmuted distributions show better flexibility to handle multi-modal data than the quadratic transmuted distributions.

Cubic transmuted Log-logistic distribution

Granzotto et al. (2017) proposed the cubic transmuted log-logistic distribution. they used the transmuted log-logistic distribution to derive the pdf of the cubic transmuted log-logistic distribution.

The pdf of the cubic transmuted log-logistic distribution is given by

$$
f_{C T L L}(x ; \gamma, \theta)=\frac{\beta x^{\beta-1} e^{\alpha}}{\left(1+e^{\alpha} x^{\beta}\right)^{4}}\left[\gamma_{1}\left(1-e^{2 \alpha} x^{2 \beta}\right)+\gamma_{2} e^{\alpha} x^{\beta}\left(2-e^{\alpha} x^{\beta}\right)+3 e^{2 \alpha} x^{2 \beta}\right], x>0
$$

The cdf corresponding to (108) is given by

$$
F_{\text {cruL }}(x ; \gamma, \theta)=\frac{x^{\beta} e^{\alpha}}{\left(1+e^{\alpha} x^{\beta}\right)}\left[\gamma_{1}+\left(\gamma_{2}-\gamma_{1}\right) \frac{x^{\beta} e^{\alpha}}{\left(1+e^{\alpha} x^{\beta}\right)}+\left(1-\gamma_{2}\right) \frac{x^{2 \beta} e^{2 \alpha}}{\left(1+e^{\alpha} x^{\beta}\right)^{2}}\right], x>0
$$

\subsubsection{The General Transmuted Family of Distributions}

Merovci et al. (2016) proposed and studied the statistical and mathematical properties of the generalized transmuted family of distributions. (Alizadeh et al. 2017) introduced a new generalized transmuted family of distributions and have described it as a linear combination of exponentiated densities in terms of the same parent distribution. Recently, Rahman et al. (2018) introduced a couple of new general transmuted family of distributions and they named k-transmuted families and they defined by

$$
F(x ; \theta)=G(x ; \theta)+[1-G(x ; \theta)] \sum_{i=1}^{k} \gamma_{i}[G(x ; \theta)]^{i}, x x \in \mathbb{R},
$$

where $\gamma_{i} \in[-1,1]$ for $i=1,2, \ldots, k$ and $-k \leq \sum_{i=1}^{k} \gamma_{i} \leq 1$, and

$$
F(x ; \theta)=G(x ; \theta)+G(x ; \theta) \sum_{i=1}^{k} \gamma_{i}[G(x ; \theta)]^{i}, x x \in \mathbb{R},
$$

where $\gamma_{1} \in[-1,1]$ and $\gamma_{i} \in[-1,1]$ for $i=1,2, \ldots, k$.

AL-Kadim (2018) introduced a generalized family of transmuted distribution which turned out to be a special case of family (110).

\subsubsection{The Weibull-G Family of Distributions}

Alzaatreh et al. (2013) and Bourguignon et al. (2014) proposed the Weibull-G family of continuous probability distributions. Weibull-G family is an interesting technique of inducting an extra shape parameter(s) to an existing $G$ distribution. Considering the cdf of the Weibull distribution which is given by

$$
F(x)=1-e^{-\alpha x^{\beta}}, x>0
$$

where $\alpha, \beta>0$ are the unknown parameters of the Weibull distribution.

They defined the cdf of the Weibull-G family by replacing $x$ with $G(x ; \theta) / S(x ; \theta)[S(x ; \theta)=1-G(x ; \theta)$, ], the cdf is given by

$$
F(x ; \alpha, \beta, \theta)=\int_{0}^{\frac{G(x ; \theta)}{1-G(x ; \theta)}} \alpha \beta t^{\beta-1} e^{-\alpha t^{\beta}} d t=1-\exp \left\{-\alpha\left[\frac{G(x ; \theta)}{S(x ; \theta)}\right]^{\beta}\right\}, x \in D \subseteq \mathbb{R} ; \alpha, \beta>0,
$$

Where $G(x ; \theta)$ is the baseline cdf, which depends on a parameter vector $\boldsymbol{\theta}^{\prime}=(\alpha, \beta)$.

The pdf corresponding to (113) is given by 


$$
f(x ; \alpha, \beta, \theta)=\alpha \beta g(x ; \theta) \frac{G(x ; \theta)^{\beta-1}}{S(x ; \theta)^{\beta+1}} \exp \left\{-\alpha\left[\frac{G(x ; \theta)}{S(x ; \theta)}\right]^{\beta}\right\},
$$

The hazard rate function of the Weibull-G family is given by

$$
h(x ; \alpha, \beta, \theta)=\frac{\alpha \beta g(x ; \theta) G(x ; \theta)^{\beta-1}}{S(x ; \theta)^{\beta+1}}=\frac{\alpha \beta G(x ; \theta)^{\beta-1}}{S(x ; \theta)^{\beta}} h(x ; \theta),
$$

whereh $(x ; \theta)=g(x ; \theta) / S(x ; \theta)$. the multiplying quantity $\alpha \beta G(x ; \theta)^{\beta-1} / S(x ; \theta)^{\beta+1}$ works as a corrected factor for the failure rate function of the parent (or baseline) distribution.

The Weibull-G family can deal with general situations in modeling and analysing time-to-event data with different shapes of the hazard (or failure) rate function. For example, if the baseline distribution is a log-logistic distribution the

Weibull-Log-logistic Distribution

$$
\frac{G(x ; \theta)^{\beta-1}}{S(x ; \theta)^{\beta+1}}=\left[1+\left(\frac{x}{S}\right)^{c}\right]^{-1}, x>0
$$

Oluyede et al. (2016)proposed the log-logistic Weibull distribution and they applied it into a lifetime data. Considering a series system and assuming that the lifetime of the component follow the log-logistic and Weibull distribution with survival functions $S_{1}(t ; \theta)=\left[1+\left(\frac{t}{s}\right)^{c}\right]^{-1}$, and $S_{2}(t ; \theta)=e^{-\alpha t^{\beta}}$, respectively. The survival $S(t)=P(T>t)$ of the system is given by

$$
S(t)=\prod_{i=1}^{2} S_{i}(t)
$$

The cdf of the log-logistic-Weibull distribution is given by

$$
F(x)=1-\left(1+\left(\frac{x}{S}\right)^{c}\right)^{-1} e^{-\alpha x^{\beta}}
$$

The corresponding pdf is given by

$$
f(x)=e^{-\alpha x^{\beta}}\left[1+\left(\frac{x}{s}\right)^{c}\right]^{-1}\left\{\alpha \beta x^{\beta-1}+\frac{c x^{c-1}}{\left(s^{c}+x^{c}\right)}\right\}
$$

Where $s, c, \alpha, \beta>0$ and $x \geq 0$.

\subsubsection{The Exponentiated Generalized Family of Distributions}

Cordeiro et al. (2013) proposed a new family of distributions, called the exponentiated generalized (EG) class of distributions. This method belongs to the parameter induction method, where the purpose is to add two new extra shape parameters to the baseline (or parameter) distribution.

The cdf of the EG class of distribution is given by

$$
F_{E G}(x ; \theta)=\left\{1-[1-G(x)]^{a}\right\}^{b}, x \in \mathbb{R},
$$

where $a>0$, and $b>0$ are two extra parameters whose role is to govern the skewness and create distributions with heavier/lighter tails.

The pdf corresponding to (120) is given by

$$
f_{E G}(x ; \theta)=a b[1-G(x)]^{a-1}\left\{1-[1-G(x)]^{a}\right\}^{b-1} g(x), x \in \mathbb{R},
$$

where $g(x)=\frac{d G(x)}{d x}$ is the pdf of the baseline (or parent) distribution. the two parameters in (121) can add entropy to the center of the Exponentiated Generalized density or possible control both tail weights.

Exponentiated generalized log-logistic distribution

Lima and Cordeiro (2017) studied the mathematical and statistical properties of a new four-parameter survival model applying the exponentiated generalized (EG) class. 
The cdf of the new distribution is given by

$$
F_{E G L L}(x ; \theta)=\left\{1-\left[1-\frac{x^{\beta}}{\alpha^{\beta}+x^{\beta}}\right]^{a}\right\}^{b}, x \in \mathbb{R},
$$

The pdf corresponding to (122) is given by

$$
f_{E G L L}(x ; \theta)=\frac{a b \beta\left(\frac{x}{\alpha}\right)^{\beta-1}}{\alpha\left[1+\left(\frac{x}{\alpha}\right)^{\beta}\right]^{2}}\left[1-\frac{x^{\beta}}{\alpha^{\beta}+x^{\beta}}\right]^{a-1}\left\{1-\left[1-\frac{x^{\beta}}{\alpha^{\beta}+x^{\beta}}\right]^{a}\right\}^{b-1}, x \in \mathbb{R},
$$

The hazard rate function of the EGLL is given by

$$
h_{E G L L}(x ; \theta)=\frac{a b \beta\left(\frac{x}{\alpha}\right)^{\beta-1}\left[1-\frac{x^{\beta}}{\alpha^{\beta}+x^{\beta}}\right]^{a-1}\left\{1-\left[1-\frac{x^{\beta}}{\alpha^{\beta}+x^{\beta}}\right]^{a}\right\}^{b-1}}{\alpha\left[1+\left(\frac{x}{\alpha}\right)^{\beta}\right]^{2}\left\{1-\left[1-\frac{x^{\beta}}{\alpha^{\beta}+x^{\beta}}\right]^{a}\right\}^{b}}, x \in \mathbb{R},
$$

The quantile function of EGLL is given by inverting (122)

$$
x_{q}=\frac{\alpha^{\beta}\left\{1-\left[1-q^{\bar{\beta}}\right]^{\frac{1}{\alpha}}\right\}}{\left[1-q^{\frac{1}{\beta}}\right]^{\frac{1}{\alpha}}}, x \in \mathbb{R},
$$

\subsubsection{The T-X Family of Distributions}

Alzaatreh et al. (2013) proposed a new family of distributions, called the T-X family of distributions as an extension to the beta-G family of distributions introduced by (Eugene et al., 2002).

If the cdf of the baseline distribution is $G(x ; \theta)$ then the cdf of the T-X family is given as

$$
F(x)=\int_{a}^{W F(x)} r(t) d t=\{R W(F(x))\},
$$

where $\mathrm{R}$ is the cdf of the baseline random variable, and the function $W(F(x)$ must satisfy the following conditions:

1. $W(F(x) \in[a, b]$,

2. $W$ is differentiable and monotonically non-decreasing

3. $W(F(x) \rightarrow a$ as $x \rightarrow-\infty$ and $W(F(x) \rightarrow b$ as $x \rightarrow \infty$

Where $[a, b]$ is the support of the baseline random variable for $-\infty \leq a<b \leq \infty$.

The pdf corresponding to (126) if it exists is given by

$$
f(x) \rightarrow\left\{\frac{d}{d x} W F(x)\right\} r\{W(F(x))\} .
$$

Alzaatreh and Ghosh (2015) introduced three sub-families of the T-X family which are beta-exponential-X family, gamma-X family, and the Weibull-X family. These three sub-families demonstrate that the T-X family consists of many sub-classes of distributions within each sub-family, one can introduce many new distributions as well as relateits members to many existing probability distributions. More details about the T-X family of distributions and their modifications we can refer to (Nasiru, 2018).

\subsubsection{The Weibull-X Family of Distributions}

Alzaatreh and Ghosh (2015) proposed the Weibull-X family of distributions. Using the concept of T-X family of distributions introduced by Alzaatreh et al. (2013).

The cdf and the pdf of the T-X family of distributions are given by; 


$$
\begin{gathered}
F(x)=\int_{a}^{-\log [1-G(x)]} r(t) d t \\
f(x) \rightarrow \frac{g(x)}{1-G(x)} r\left\{-\log [1-G(x)]=h_{g}(x) r H_{f}(x) .\right.
\end{gathered}
$$

Where $h_{g}(x)$ and $H_{f}(x)$ are the hazard rate and the cumulative hazard rate functions associated with $g(x)$.

If a random variable $\mathrm{T}$ follows the Weibull distribution with a pdf of

$$
r(t)=\left(\frac{\beta}{\alpha}\right)\left(\frac{t}{\alpha}\right) \beta-1 e^{-\left(\frac{t}{\alpha}\right)^{\beta}, t>0}
$$

The pdf and cdf of the Weibull-X family of distributions is given by

$$
\begin{aligned}
& f(x) \rightarrow \frac{\beta g(x)}{\alpha(1-G(x))}\left(\frac{-\log [1-G(x)]}{\alpha}\right)^{\beta-1} \exp \left\{-\left(\frac{-\log [1-G(x)]}{\alpha}\right){ }^{\beta}\right\} \\
& F(x)=1-\exp \left\{-\left(\frac{-\log [1-G(x)]}{\alpha}\right) \beta\right\}, x \in \mathbb{R}
\end{aligned}
$$

\subsubsection{The Exponentiated Kumaraswamy-G family}

Lemonte et al. (2013)proposed the exponentiated Kumaraswamy-G family of distributions. The cdf and the pdf of the EK family are given by

$$
\begin{gathered}
F(x ; \theta)=\left[1-\left(1-x^{\alpha}\right)^{\beta}\right]^{\gamma}, x \in(0,1) \\
f(x ; \theta)=\alpha \beta \gamma x^{\alpha-1}\left(1-x^{\alpha}\right)^{\beta-1}\left[1-\left(1-x^{\alpha}\right)^{\beta-1}\right]^{\gamma-1}, x \in(0,1)
\end{gathered}
$$

where $\alpha \beta$ and $\gamma$ are extra positive shape parameters. The distribution (134) provides more options for analysing data restricted to the interval $(0,1)$.

\subsubsection{The Generalized Weibull-G Family of Probability Distributions}

Cordeiro et al. (2015)proposed a new generalized Weibul-G family of probability distributions and they studied the mathematical and statistical properties of the family and some special models.

The cdf of the generalized Weibull family is given by

$$
F(x ; \alpha, \beta, \theta)=\alpha \beta \int_{0}^{-\log [1-G(x ; \theta)]} t^{\alpha-1} e^{-\alpha t^{\beta}} d t=1-\exp \left\{-\alpha(-\log [1-G(x ; \theta)])^{\beta}\right\}
$$

The pdf corresponding to (130) is given by

$$
f(x ; \alpha, \beta, \theta)=\frac{\alpha \beta g(x ; \theta)}{[1-G(x ; \theta)]}\{-\log [1-G(x ; \theta)]\}^{\beta-1} \exp \left\{-\alpha(-\log [1-G(x ; \theta)])^{\beta}\right\}
$$

The generalized Weibull-Log-logistic Distribution

Cordeiro et al. (2015) introduced the generalized Weibull log-logistic distribution (GWLL).

the pdf of the GWLL with a log-logistic distribution having a scale parameter of a and a shape parameter of $b$ is given by;

$$
f(x)=\frac{\alpha \beta a x^{b-1}}{b^{a}}\left(1+\left(\frac{x}{b}\right)^{a}\right)^{-1}\left\{\log \left(1+\left(\frac{x}{b}\right)^{a}\right)\right\}^{\beta-1} \exp \left\{-\alpha\left(\log \left[\left(1+\left(\frac{x}{b}\right)^{a}\right)\right]\right)^{\beta}\right\}
$$

The literature on the generalized log-logistic distribution using generator (or parameter induction) methods is enrich enough and is also rapidly improving. We now describe the generalization of the log-logistic distribution using compounding and other methods in the following.

\subsubsection{The Gamma Uniform Family of Probability Distributions}

Torabi and Hedesh (2016) proposed a new generator approach called the gamma-uniform family of distributions. the cdf of the family is given by

$$
F(x)=\alpha \beta \int_{0}^{\frac{x-a}{b-x}} \frac{e^{-\frac{w}{\beta}} w^{\alpha-1}}{\Gamma(\alpha) \beta^{\alpha}} d w=1-\mathrm{Q}\left\{\alpha \frac{x-a}{\beta(b-x)}\right\}, a<x<b .
$$


The pdf and hazard rate function corresponding to (138) are given by

$$
\begin{aligned}
& f(x)=\frac{(b-a) e^{-\frac{x-a}{\beta(b-x)}}\left(\frac{x-a}{b-x}\right)^{\alpha-1}}{(\mathrm{~b}-\mathrm{x})^{2} \Gamma(\alpha) \beta^{\alpha}}, a<x<b \\
& h(x)=\frac{(b-a) e^{-\frac{x-a}{\beta(b-x)}\left(\frac{x-a}{b-x}\right)^{\alpha-1}}}{(\mathrm{~b}-\mathrm{x})^{2} \Gamma\left(\alpha, \frac{x-a}{\beta(b-x)}\right) \beta^{\alpha}}, a<x<b
\end{aligned}
$$

This distribution can be applied for modeling any data set with changing the extra parameters.

\subsection{Compounding Methods}

The compounding method is a technique that combine two or more existing distributions 4.2.1 The Exponentiated G-Geometric Class

Nadarajah et al. (2015)introduced the exponentiated G-geometric also known as the generalized G-geometric class. If $T[G(x ; \tau) ; \gamma]=G(x ; \tau)^{\gamma}$ and $\mathrm{N}$ be a geometric random variable with failure probability parameter $p \in(0,1)$ and probability mass function $\mathbb{P}(N=n)=q p^{n-1}, n=1,2, \ldots, p$. where $\mathrm{p}$ is the success probability.

If we define $X=\min \left(Y_{1}, \ldots, Y_{N}\right)$, the unconditional of cdf of the exponentiated G-geometric (EGG) class can follow as

$$
F_{E G G}(x ; \theta)=\sum_{n=1}^{\infty}(1-p) p^{n-1}\left[1-\left(1-G(x ; \tau)^{\gamma}\right)^{n}\right]=\frac{G(x ; \tau)^{\gamma}}{\left\{1-p\left[1-G(x ; \tau)^{\gamma}\right]\right\}}
$$

Where $\gamma>0,0<p<1$ are two extra shape parameters. This new family can also be obtained by modification or an extension of Marshall-Olkin family of distribution by replacing the baseline CDF with its exponentiation and adding two more extra shape parameters to the original distribution.

The pdf corresponding to (141) is given by

$$
f_{E G G}(x ; \theta)=\frac{\gamma(1-p) G^{\gamma-1}(x ; \tau) g(x ; \tau)}{\left\{1-p\left[1-G(x ; \tau)^{\gamma}\right]\right\}^{2}}
$$

The exponentiated log-logistic geometric distribution

Mendoza et al. (2016)proposed and studied the exponentiated log-logistic geometric distribution. they derived some of the mathematical and statistical properties of the EEG Log-logistic distribution.

If $\left\{Y_{i}\right\}_{i=1}^{Z}$ are an i.i.d random variables having the exponentiated log-logistic distribution with cdf and pdf

$$
\begin{gathered}
F_{E L L}(x)=P_{E L L}(\{y \mid y \leq x\})=\left[\frac{\left(\frac{x}{\alpha}\right)^{\beta}}{1+\left(\frac{x}{\alpha}\right)^{\beta}}\right]^{\gamma} \text { for } x \geq 0 \\
f_{E L L}(x)=\frac{\beta \gamma\left(\frac{x}{\alpha}\right)^{\beta \gamma-1}}{\alpha\left(1+\left(\frac{x}{\alpha}\right)^{\beta}\right)^{\gamma+1}} \text { for } x \geq 0
\end{gathered}
$$

where $\alpha, \beta$, and $\gamma>0$ are the unknown parameters of the model. For $\gamma=1$, we obtain as a special case the LL distribution.

Then,

- The conditional density function of EGG log-logistic function for $X_{1}=\min \left(\left\{Y_{i}\right\}_{i=1}^{Z}\right)$ : is

$$
f(x \mid z ; \gamma, \alpha, \beta)=\frac{z \beta \gamma}{\alpha}\left(\frac{x}{\alpha}\right)^{\beta \gamma-1}\left[1+\left(\frac{x}{\alpha}\right)^{\beta}\right]^{-(\gamma+1)}\left\{1-\left[1+\left(\frac{x}{\alpha}\right)^{-\beta}\right]^{-\gamma}\right\}^{z-1}
$$

The pdf of the exponentiated log-logistic geometric type I (ELLGI) distribution reduces to 


$$
f_{E L L G I}(x ; \theta)=\frac{(1-p) \beta \gamma}{\alpha}\left(\frac{x}{\alpha}\right)^{\beta \gamma-1}\left[1+\left(\frac{x}{\alpha}\right)^{\beta}\right]^{-(\gamma+1)}\left\{1-p\left[1-\left\{1+\left(\frac{x}{\alpha}\right)^{-\beta}\right\}^{-\gamma}\right]\right\}^{-2}
$$

Where $x>0, \theta$ is the vector of parameters $((\alpha, \beta, \gamma>0$, and $p \in(0,1)$.

The cdf corresponding to (146) is given by

$$
F_{E L L G I}(x ; \theta)=\left[1+\left(\frac{x}{\alpha}\right)^{-\beta}\right]^{-\gamma}\left\{1-p\left[1-\left\{1+\left(\frac{x}{\alpha}\right)^{-\beta}\right\}^{-\gamma}\right]\right\}^{-1}, x>0
$$

The hazard rate function corresponding to (146) is given by

$$
\begin{gathered}
h_{E L L G I}(x ; \theta)=\frac{(1-p) \beta \gamma}{\alpha}\left(\frac{x}{\alpha}\right)^{\beta \gamma-1}\left[1+\left(\frac{x}{\alpha}\right)^{\beta}\right]^{-(\gamma+1)}\left\{1-p\left[1-\left\{1+\left(\frac{x}{\alpha}\right)^{-\beta}\right\}^{-\gamma}\right]\right\}^{-1} \\
\times\left\{1-p\left[1-\left\{1+\left(\frac{x}{\alpha}\right)^{-\beta}\right\}^{-\gamma}\right]-\left\{1+\left(\frac{x}{\alpha}\right)^{-\beta}\right\}^{-\gamma}\right\}^{-1}, x>0
\end{gathered}
$$

- The conditional density function of EGG log-logistic function for $X_{2}=\max \left(\left\{Y_{i}\right\}_{i=1}^{Z}\right)$ : is

$$
f(x \mid z ; \gamma, \alpha, \beta)=\frac{z \beta \gamma}{\alpha}\left(\frac{x}{\alpha}\right)^{\beta \gamma-1}\left[1+\left(\frac{x}{\alpha}\right)^{\beta}\right]^{-(\gamma+1)}\left[1+\left(\frac{x}{\alpha}\right)^{-\beta}\right]^{-\gamma(z-1)}
$$

The pdf of the exponentiated log-logistic geometric type II (ELLGII) distribution reduces to

$$
f_{\text {ELLGII }}(x ; \theta)=\frac{(1-p) \beta \gamma}{\alpha}\left(\frac{x}{\alpha}\right)^{\beta \gamma-1}\left[1+\left(\frac{x}{\alpha}\right)^{\beta}\right]^{-(\gamma+1)}\left\{1-p\left[1+\left(\frac{x}{\alpha}\right)^{-\beta}\right]^{-\gamma}\right\}^{-2}
$$

Where $x>0, \theta$ is the vector of parameters $((\alpha, \beta, \gamma>0$, and $p \in(0,1)$.

The cdf corresponding to (150) is given by

$$
F_{E L L G I I}(x ; \theta)=(1-p)\left[1+\left(\frac{x}{\alpha}\right)^{-\beta}\right]^{-\gamma}\left\{1-p\left[1+\left(\frac{x}{\alpha}\right)^{-\beta}\right]^{-\gamma}\right\}^{-1}, x>0
$$

The hazard rate function corresponding to (150) is given by

$$
h_{E L L G I}(x ; \theta)=\frac{\frac{(1-p) \beta \gamma}{\alpha}\left(\frac{x}{\alpha}\right)^{\beta \gamma-1}\left[1+\left(\frac{x}{\alpha}\right)^{\beta}\right]^{-(\gamma+1)}\left\{1-p\left[1+\left(\frac{x}{\alpha}\right)^{-\beta}\right]^{-\gamma}\right\}^{-2}}{1-(1-p)\left[1+\left(\frac{x}{\alpha}\right)^{-\beta}\right]^{-\gamma}\left\{1-p\left[1+\left(\frac{x}{\alpha}\right)^{-\beta}\right]^{-\gamma}\right\}^{-1}}
$$

\subsection{Other Methods}

In this section, we present some new techniques for generating new probability distributions.

\subsubsection{Khan and Khosa's Generalized Log-Logistic Distribution}

Khan and Khosa (2015) proposed a generalized log-logistic distribution that belongs to the PH family and they described that it has properties identical to those of log-logistic, and tend to the Weibull in the limit, and they defined that these features enable the model to handle all kinds of hazard functions.

The pdf, survivor function, hazard rate function, and the cumulative hazard rate function of the Generalized log-logistic distribution is given by respectively;

$$
\begin{aligned}
& f(t ; \theta)=\frac{k \rho(\rho t)^{k-1}}{\left(1+(\gamma t)^{k}\right)^{2}}, t>0, \\
& S(t ; \theta)=\left[1+(\gamma t)^{k}\right]^{-\frac{p^{k}}{\gamma^{k}}}, \\
& h(t ; \theta)=\frac{k \rho(\rho t)^{k-1}}{1+(\gamma x)^{k}} \\
& H(t ; \theta)=\frac{p^{k}}{\gamma^{k}} \log \left[1+(\gamma t)^{k}\right],
\end{aligned}
$$

\section{Estimation of the Parameters}

In the statistics literature, due to the importance of the LL distribution and its generalizations, the estimation of the 
unknown parameters has been widely studied from the two main inferential statistics of thought: frequentists and Bayesians. From the frequentist approach, there are several different techniques that were proposed for parameter estimation but the maximum likelihood estimators (MLEs) are commonly used in most of the studies because of their appealing properties and can be applied when building confidence regions and intervals and also in test statistics. Although MLE method has been proven to be consistent, asymptotically efficient under very general conditions, it was found that it breaks down as one parameter tending to cause the likelihood to be infinite, rendering the other parameters inconsistent and is not applicable to J-shape distributions (Cheng and Amin, 1983). Ranneby, (1984) mentioned that the MLE is unbound and inefficient in the estimation of the mixtures of continuous distributions and heavy-tailed distributions. Therefore, to overcome the drawbacks in the MLE method (Cheng and Amin, 1979) proposed a maximum product of spacings (MPS) estimator to deal with those problems as it will return valid results over a much wider range of distributions and their generalizations. The method was also developed independently by (Ranneby, 1984).

In this study, we applied the maximum product spacings (MPS) method to estimate the unknown parameters for most of the generalized distributions discussed in this paper. For more information about the MPS estimator we can refer to (Cheng and Amin, 1979, 1983; Kawanishi, 2020; Ranneby, 1984; Thongkairat et al. 2018). The MPS estimator is a general technique used for estimating unknown parameters from observations with continuous univariate distributions and is an alternative to the MLE method. The log-likelihood for the distribution parameters can be maximized by using nonlinear likelihood equations obtained by differentiating the log-likelihood or by using software programs and packages. In this study, we applied the MPS (Maximum Product Spacing) package (version 2.3.1) (Teimouri, 2018) available in the $\mathrm{R}$ programming language to estimate the unknown parameters of the generalized distributions. The package has been continuously updated and more information can be obtained from https://cran.rstudio.com/web/packages/MPS/index.html. Currently, Bayesian inference of the log-logistic parameters and some of its generalizations has also received attention in the literature and some of them are still needs to be studied.

\section{Real-life Data Application}

In this section, we compare the performances of some of the generalizations of the log-logistic distribution in Section 5 using a real-life data set. The data represent the survival times of 121 patients with breast cancer obtained from a large hospital in a period from 1929 to 1938 (Lee and Wang, 2003). The data are:

$(154.0,139.0,129.0,129.0,127.0,126.0,125.0,117.0,115.0,111.0,109.0,109.0,105.0,103.0,96.0,93.0,90.0$,

89.0, 88.0, 83.0, 80.0, 78.0, 69.0, 68.0, 67.0, 67.0, 65.0, 65.0, 62.0, 61.0, 60.0, 60.0, 60.0, 59.0, 58.0, 57.0, 56.0,

55.0, 54.0, 52.0, 51.0, 51.0, 51.0, 49.0, 48.0, 47.0, 46.0, 46.0, 45.0, 45.0, 44.0, 43.0, 43.0, 43.0, 42.0, 41.0, 41.0,

41.0, 40.0, 40.0, 40.0, 39.0, 39.0, 38.0, 38.0, 38.0, 37.0, 37.0, 37.0, 35.0, 35.0, 32.0, 31.0, 31.0, 30.0, 29.1, 28.2,

27.9, 24.0, 24.0, 23.6, 23.4, 23.0, 21.1, 21.0, 21.0, 20.9, 20.4, 19.8, 17.9, 17.5, 17.3, 17.2, 16.8, 16.5, 16.3, 16.2,

$15.7,15.5,14.8,14.4,14.4,13.5,12.3,12.2,11.8,11.0,10.3,8.4,8.4,7.5,7.4,6.8,6.6,6.3,6.2,5.6,5.0,4.0$,

$0.3,0.3)$

Table 1. Descriptive statistics of the data

\begin{tabular}{ccccccc}
\hline Mean & Median & Variance & Skewness & Kurtosis & Minimum & Maximum \\
\hline 46.33 & 40 & 1244.464 & 1.03 & 0.35 & 0.3 & 154 \\
\hline
\end{tabular}

We fitted the following scale-shape variations of some of the surveyed distributions: The beta log-logistic distribution (BLL), the Kumaraswamy log-logistic distribution (KWLL), the exponentiated log-logistic distribution (ELL), the exponentiated generalized log-logistic distribution (EGLL), the Zografos-Balakrishnan log-logistic distribution (ZBLL), the Marshall-Olkin log-logistic distribution (MO-LL), the Weibull-log-logistic distribution (WLL), the Weibull-X (T-X) log-logistic distribution (WXLL), the exponentiated Kumaraswamy log-logistic distribution (EKWLL), the gamma-uniform log-logistic distribution (GLL), and the Log-logistic (LL) distribution.

Each distribution was fitted by the method of maximum product spacings (MPS). Table 2 gives the values of the MPS estimates of the model parameters for the BLL, KWLL, ELL, ZBLL, EGLL, MO-LL, WLL, WXLL, EKWLL, GLL, and LL models fitted to the exceedances of breast cancer data. We estimate the unknown parameters of each model by maximum product spacings (MPS). There exist many maximization methods in R packages like NM (Nelder-Mead), BFGS (Broyden-Fletcher Goldfarb-Shanno), NR (Newton-Raphson), BHHH (Berndt-Hall-Hall-Hausman), and SANN (Simulated-Annealing) methods. In this study, the maximum product spacing estimators (MPS) are computed using Nelder-Mead optimization (NM) and the measures of goodness of fit AIC, BIC, CAIC, HQIC, Anderson-Darling (A*) 
and Cramer-von Misses ( $\mathrm{W}^{*}$ ) are used to compare the ten selected models.

Table 2. MPS estimators of the model parameters and the maximum of the log-likelihood function

\begin{tabular}{ccccccc}
\hline Distributions & \multicolumn{7}{c}{ MPS estimators of the parameters } \\
\hline GLL $(\mathrm{a}, \alpha, \beta)$ & 0.765 & $\mathbf{b}$ & $\mathbf{c}$ & $\boldsymbol{\alpha}$ & $\boldsymbol{\beta}$ & $\boldsymbol{\ell}(\widehat{\boldsymbol{\theta}})$ \\
\hline WXLL $(\mathrm{a}, \mathrm{b}, \alpha, \beta)$ & 0.878 & 0.865 & & 1.495 & 63.618 & $\mathbf{- 5 7 9 . 0 1 1}$ \\
KWLL $(\mathrm{a}, \mathrm{b}, \alpha, \beta)$ & 0.366 & 4.311 & 1.439 & 44.828 & -579.143 \\
EGLL $(\mathrm{a}, \mathrm{b}, \alpha, \beta)$ & 6.870 & 0.691 & & 3.113 & 207.018 & -579.211 \\
WLL $(\mathrm{a}, \mathrm{b}, \alpha, \beta)$ & 14.442 & 0.610 & & 0.115 & 222.676 & -579.411 \\
BLL $(\mathrm{a}, \mathrm{b}, \alpha, \beta)$ & 0.696 & 6.885 & & 1.661 & 203.623 & -579.412 \\
ZBLL $(\mathrm{a}, \alpha, \beta)$ & 0.351 & & & 3.011 & 79.117 & -580.609 \\
ELL $(\mathrm{a}, \alpha, \beta)$ & 0.319 & & & 3.305 & 71.682 & -580.748 \\
EKWLL $(\mathrm{a}, \mathrm{b}, \mathrm{c}, \alpha, \beta)$ & 3.603 & 5.835 & 0.181 & 1.700 & 82.094 & -579.330 \\
MO-LL $(\mathrm{a}, \alpha, \beta)$ & 1.137 & & & 1.800 & 32.683 & $\mathbf{- 5 8 7 . 6 7 7}$ \\
LL $(\alpha, \beta)$ & & & & 1.856 & 35.177 & -587.599 \\
\hline
\end{tabular}

Table 3 lists the values of the following statistics: Akaike Information Criterion (AIC), values of Bayesian Information Criterion (BIC), values of Consistent Akaike Information Criterion (CAIC), values of Hannan-Quinn information Criterion (HQIC), Cramer-von Misses statistic ( $W^{*}$ ), and Anderson-Darlin Statistic (A*). The smaller the values of these criteria the better the fit. For more information about these criteria, we refer to (Anderson \& Burnham, 2004; Bierens, 2004; Burnham \& Anderson, 2004)and (Fang, 2011).

Table 3. Goodness-of-fit tests for the generalizations of the 2-parameter log-logistic distribution

\begin{tabular}{ccccccc}
\hline Distribution & \multicolumn{7}{c}{ Goodness of fit (G-O-F) criteria } \\
\hline BLL & $\mathbf{1 1 7 2 . 4 1 1}$ & $\mathbf{1 1 6 4 . 0 2 3}$ & $\mathbf{1 1 6 4 . 2 2 9}$ & $\mathbf{1 1 6 7 . 4 3 0}$ & 0.463 & 0.063 \\
WXLL & 1177.469 & 1166.286 & 1166.631 & 1170.828 & 0.407 & 0.055 \\
KWLL & 1177.607 & 1166.424 & 1166.769 & 1170.966 & 0.538 & 0.079 \\
EGLL & 1177.786 & 1166.602 & 1166.947 & 1171.144 & 0.422 & 0.057 \\
WLL & 1178.006 & 1166.823 & 1167.168 & 1171.365 & 0.417 & 0.057 \\
BLL & 1178.008 & 1166.825 & 1167.170 & 1171.367 & 0.431 & 0.058 \\
ZBLL & 1175.606 & 1167.218 & 1167.423 & 1170.625 & 0.442 & 0.052 \\
ELL & 1175.885 & 1167.498 & 1167.703 & 1170.904 & 0.439 & 0.051 \\
EKWLL & 1182.639 & 1168.660 & 1169.182 & 1174.338 & 0.476 & 0.062 \\
LL & 1184.791 & 1179.199 & 1179.301 & 1181.47 & $\mathbf{1 . 2 5 7}$ & $\mathbf{0 . 2 0 9 6}$ \\
MO-LL & $\mathbf{1 1 8 9 . 7 4 2}$ & $\mathbf{1 1 8 1 . 3 5 5}$ & $\mathbf{1 1 8 1 . 5 6 0}$ & $\mathbf{1 1 8 4 . 7 6 1}$ & $\mathbf{1 . 0 8 4}$ & $\mathbf{0 . 1 3 1}$ \\
\hline
\end{tabular}

We can see that the 3-parameter gamma-uniform log-logistic distribution gives the smallest AIC, CAIC, BIC, and HQIC values. The GLL distribution provides significantly better fits than all of the other distributions, including the WELL and other distributions. MO-LL and LL distributions give the largest values for all criterion and tests, also the EKWLL distribution, these distributions may be thought to give worst fits. The maximum likelihood estimates of the best fitting GLL distribution are $\hat{\alpha}=0.7652255, \widehat{\beta}=1.4947180, \hat{\gamma}=63.6183351$. The fitting was performed using the $\mathrm{R}$ package MPS (Teimouri, 2018).

Shifted log-logistic distribution or generalized log-logistic distribution or simply a three-parameter log-logistic distribution is an extension of the 2-parameter log-logistic distribution by an adding a shift parameter or location parameter. If we apply the generalization of the shifted log-logistic distribution by adding an extra parameter(s) and then apply the above breast cancer data. The comparison of the selected models plus the Shifted (3-parameter) Log-logistic distribution (SLL) are listed in Table 4. 
The pdf of the shifted log-logistic distribution is given by

$$
f(x)=\frac{\frac{\beta}{\alpha}\left(\frac{x-\mu}{\alpha}\right)^{\beta-1}}{\left(1+\left(\frac{x-\mu}{\alpha}\right)^{\beta}\right)^{2}},
$$

Table 4. Goodness-of-fit tests for the generalizations of the shifted log-logistic distribution

\begin{tabular}{|c|c|c|c|c|c|c|}
\hline \multirow[t]{2}{*}{ Distribution } & \multicolumn{5}{|c|}{ Goodness of fit (G-O-F) criteria } & \multirow[b]{2}{*}{$\mathbf{W}^{*}$} \\
\hline & BIC & AIC & CAIC & HQIC & $\mathbf{A}^{*}$ & \\
\hline GLL & 1178.489 & 1167.306 & 1167.650 & 1171.848 & 0.352 & 0.051 \\
\hline WXLL & 1182.851 & 1168.872 & 1169.394 & 1174.549 & 0.411 & 0.059 \\
\hline WLL & 1183.550 & 1169.572 & 1170.093 & 1175.249 & 0.365 & 0.052 \\
\hline KWLL & 1183.807 & 1169.828 & 1170.350 & 1175.506 & 0.372 & 0.051 \\
\hline ZBLL & 1181.69 & 1170.506 & 1170.851 & 1175.048 & 0.410 & 0.051 \\
\hline ELL & 1181.954 & 1170.771 & 1171.116 & 1175.313 & 0.412 & 0.051 \\
\hline BLL & 1185.310 & 1171.331 & 1171.331 & 1177.009 & 0.395 & 0.062 \\
\hline EGLL & 1185.337 & 1171.358 & 1171.880 & 1177.035 & 0.385 & 0.052 \\
\hline EKWLL & 1188.942 & 1172.168 & 1172.904 & 1178.941 & 0.437 & 0.054 \\
\hline SLL & 1183.224 & 1174.836 & 1175.042 & 1178.243 & 0.795 & 0.130 \\
\hline MO-LL & 1188.349 & 1177.165 & 1177.510 & 1181.707 & 0.606 & 0.085 \\
\hline LL & 1184.791 & 1179.199 & 1179.301 & 1181.470 & 1.257 & 0.2096 \\
\hline
\end{tabular}

We can see that the 4-parameter gamma-uniform log-logistic distribution gives the smallest AIC, CAIC, BIC, HQIC, A*, and $\mathrm{W}^{*}$ values. The GLL distribution provides significantly better fits than all of the other distributions, including the WELL and other distributions. MO-LL and LL distributions give the largest values for all criterion and tests, also the EKWLL distribution, these distributions may be thought to give worst fits. The maximum likelihood estimates of the best fitting GLL distribution are $\hat{\alpha}=1.7621529, \widehat{\beta}=0.9813133, \widehat{\gamma}=27.1799543, \widehat{\lambda}=-1.8892325$.

\section{TTT Plot}

In the survival and reliability analysis, there is a qualitative information about the failure rate shape, which can help in selecting a specified model. The Total time on test (TTT) plot or TTT transform is a device used for assessing the empirical behavior of the hazard (or failure) rate function. The hazard rate may be constant, decrease, increase, be an upside-down bathtub shaped, bathtub shaped or indicate a more complicated process. The TTT plot for the above survival data is displayed in Fig 1, which reveals an increasing hazard rate function. This plot reveals that the distributions with increasing hazard rate function could be good candidates for modeling the above cancer data. In our case, the GLL is the one that best fits the data.

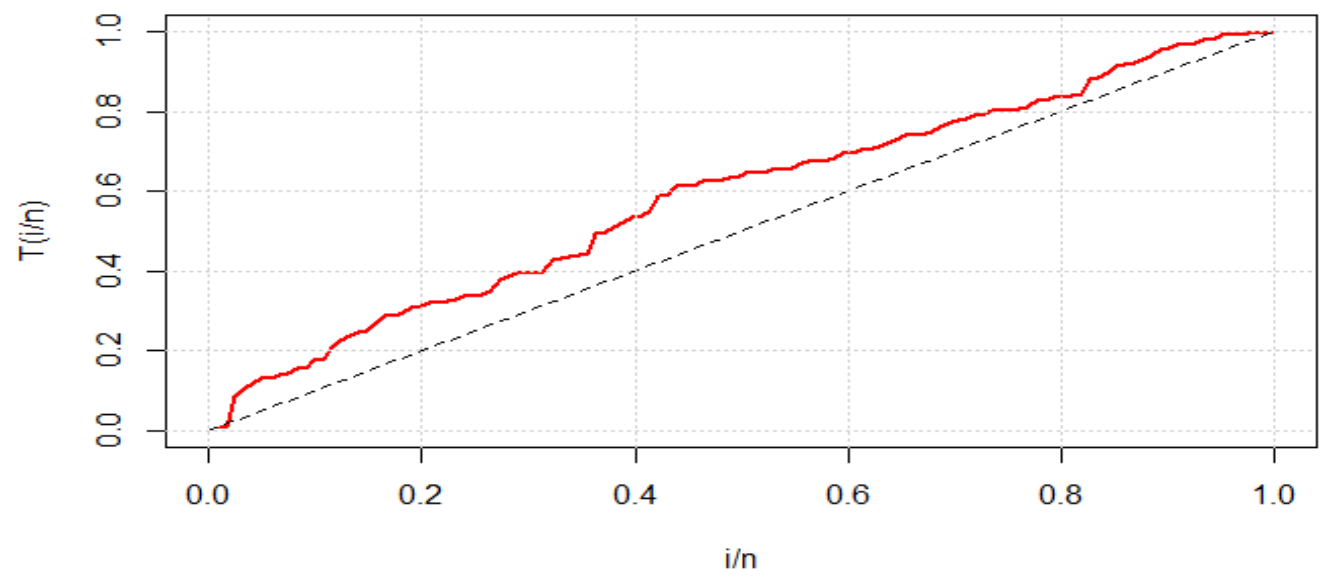

Figure 1. The TTT-plot for Cancer data 


\section{Censored Data and G-Classes of the Log-Logistic Distribution}

The generating and extension of the existing probability distributions is an essential topic in survival and reliability analysis and has been applied in many applications in fields like social sciences, biological sciences, engineering, economics, physics, chemistry, medicine among others. Extension and generating of an existing probability distribution might allow to the resulting distribution to accommodate non-monotone forms for the failure rate function. Lai et al (2013) stated that the time of failure or life can have different interpretations depending on the area applications. Therefore, we can obtain more flexible distributions for modeling this kind of random variables. On the other hand, effectiveness and tractability for modeling censored data require, among other things, closed form expressions for the cumulative distribution function.

The LL distribution has been found to be very useful for modeling and analysing the incomplete (censored and truncated) data in the area of survival and reliability analysis. The LL distribution is an effective model for censored data, especially where the hazard rate is non-monotonic (i.e. incidence of an event increases after some finite time and then slowly decreases). When it comes to the extension of the log-logistic distribution using the G-classes of distributions, the Kumaraswamy-G family of distributions can be effective and tractable models for incomplete (or censored) data. The Marshall-Olkin family and the Exponentiated-G family of distributions can also be effective and tractable models for censored data, provided $\mathrm{G}$ is in closed form. However, beta-G and Mc-G distributions may not be effective or tractable models for censored data since their cumulative distribution functions involve the incomplete beta function.

\section{Concluding Remarks}

The LL distribution is one of the most commonly used distributions in survival and reliability analysis, particularly for events where the hazard rate is non-monotonic. It has also appeared in the literature under other names, such as Fisk distribution. Various LL extensions and generalizations have been introduced in recent years. In this paper, we have listed twenty distributions obtained from different generated families and compounding methods on the LL distribution. We review some of the statistical and mathematical properties of the LL distribution. We expect that these extensions or generalizations of the LL distributions will be an addition to the art of constructing useful models and lifetime distributions in general. One can discover easier the type of G-Classes that still is not applied to the LL distribution. The generalization of log-logistic distribution through generator, compounding and other methods was first applied in the area of survival and reliability analysis. After that time, several researchers have successfully applied these methods of generalizations to model lifetime and survival data. At present, these methods are being applied in the areas of engineering, economics, environmental, medical, hydrology, social science among others to handle more complex data.

\section{Future Projects after the Survey}

We hope our work will be of value to the statistical and probability community. As for the scope of future work, the possible future projects are: (1) to propose more new extensions of the LL distribution which have not been attempted; (2) to review and extend some of the generalized LL distributions, (3) to prepare a review and new developments on parametric survival models, (4) to derive some mathematical and statistical properties of the new extended distributions, (5) to estimate the parameters of the new extensions using both the classical and Bayesian approaches; (6) to review the multi variate, matrix variate and complex variate of the generalized LL distribution; and (6) to applied the new extensions of the LL distribution into a real-life data sets.

\section{Acknowledgements}

We would like to express our heartfelt appreciation to Pan African University, Institute for Basic Sciences, Technology and Innovation (PAUSTI), and African Union (AU) Commission for their financial support during the research study. The first author would also like to thank the supervisors for their tireless effort during the study. Also, the authors would like to thank the editor and reviewers for their careful checking of the details and for the insightful comments and suggestions that made this manuscript better.

\section{References}

Ahmad, Z., Hamedani, G. G., \& Butt, N. S. (2019). Recent developments in distribution theory: A brief survey and some new generalized classes of distributions. Pakistan Journal of Statistics and Operation Research, 15(1), 87110. https://doi.org/10.18187/pjsor.v15i1.2803

AL-Kadim, K. A. (2018). $\{\mathrm{P}\}$ roposed $\{\mathrm{G}\}$ eneralized $\{\mathrm{F}\}$ ormula for $\{\mathrm{T}\}$ ransmuted $\{\mathrm{D}\}$ istribution. Journal of University of Babylon, 26(4), 66-74.

Aldahlan, M. A. (2020). Alpha Power Transformed Log-Logistic Distribution with Application to Breaking Stress Data. Advances in Mathematical Physics, 2020. https://doi.org/10.1155/2020/2193787 
Alexander, C., Cordeiro, G. M., Ortega, E. M. M., \& Sarabia, J. M. (2012). Generalized beta-generated distributions. Computational Statistics and Data Analysis, 56(6), 1880-1897. https://doi.org/10.1016/j.csda.2011.11.015

Alizadeh, M., Merovci, F., \& Hamedani, G. G. (2017). Generalized transmuted family of distributions: Properties and applications. Hacettepe Journal of Mathematics and Statistics, 46(4). https://doi.org/10.15672/HJMS.201610915478

Alshangiti, A. M., Kayid, M., \& Alarfaj, B. (2016). Further results involving Marshall-Olkin log-logistic distribution: reliability analysis, estimation of the parameter, and applications. SpringerPlus, 5(1). https://doi.org/10.1186/s40064-016-2007-X

Alzaatreh, A., \& Ghosh, I. (2015). On the Weibull-X family of distributions. Journal of Statistical Theory and Applications, 14(2), 169. https://doi.org/10.2991/jsta.2015.14.2.5

Alzaatreh, A., Lee, C., \& Famoye, F. (2013). A new method for generating families of continuous distributions. Metron, 71(1), 63-79. https://doi.org/10.1007/s40300-013-0007-y

Anderson, D., \& Burnham, K. (2004). Model selection and multi-model inference. Second. NY: Springer-Verlag, 63(2020), 10.

Aryal, G. R. (2013). Transmuted log-logistic distribution. Journal of Statistics Applications \& Probability, 2 (1), 11.

Aryal, G. R., \& Tsokos, C. P. (2009). On the transmuted extreme value distribution with application. Nonlinear Analysis, Theory, Methods and Applications, 71(12), e1401-e1407. https://doi.org/10.1016/j.na.2009.01.168

Aryal, G. R., \& Tsokos, C. P. (2011). Transmuted Weibull Distribution: A Generalization of the Weibull Probability Distribution. European Journal of Pure and Applied Mathematics, 4(2), 89-102.

Ashkar, F., \& Mahdi, S. (2003). Comparison of two fitting methods for the log-logistic distribution. Water Resources Research, 39(8).

Aslam, M., Hussain, Z., \& Asghar, Z. (2018). Cubic transmuted-g family of distributions and its properties. Stochastics and Quality Control, 33(2), 103-112.

Aslam, M., \& Jun, C.-H. (2010). A double acceptance sampling plan for generalized log-logistic distributions with known shape parameters. Journal of Applied Statistics, 37(3), 405-414.

Bacon, R. W. (1993). PRACTITIONERS CORNER: A Note on the Use of the Log-Logistic Functional Form for Modelling Saturation Effects. Oxford Bulletin of Economics and Statistics, 55(3), 355-361. https://doi.org/10.1111/j.1468-0084.1993.mp55003006.x

Barrios, R., \& Dios, F. (2012). Exponentiated Weibull distribution family under aperture averaging for Gaussian beam waves. Optics Express, 20(12), 13055-13064.

Bennett, S. (1983). Log-logistic regression models for survival data. Journal of the Royal Statistical Society: Series $C$ (Applied Statistics), 32(2), 165-171.

Bierens, H. J. (2004). Information criteria and model selection. Manuscript, Penn State University.

Bourguignon, M., Ghosh, I., \& Cordeiro, G. M. (2016). General Results for the Transmuted Family of Distributions and New Models. Journal of Probability and Statistics, 2016. https://doi.org/10.1155/2016/7208425

Bourguignon, M., Silva, R. B., \& Cordeiro, G. M. (2014). The Weibull-G Family of Probability Distributions. Journal of Data Science, 12(1), 53-68. https://doi.org/10.6339/JDS.2014.12(1).1210

BRÜEDERL, J., \& Diekmann, A. (1995). The log-logistic rate model: Two generalizations with an application to demographic data. Sociological Methods \& Research, 24(2), 158-186.

Burnham, K. P., \& Anderson, D. R. (2004). Multimodel inference: understanding AIC and BIC in model selection. Sociological Methods \& Research, 33(2), 261-304.

Casella, G., \& Berger, R. L. (2002). Statistical inference (Vol. 2). Duxbury Pacific Grove, CA.

Chaudhary, A. K. (2019). Frequentist Parameter Estimation of Two-Parameter Exponentiated Log-logistic Distribution BB. NCC Journal, 4(1), 1-8. https://doi.org/10.3126/nccj.v4i1.24727

Chaudhary, A. K., \& Kumar, V. (2014). Bayesian Estimation of Three-Parameter Exponentiated Log-logistic Distribution, 9(2), 66-81.

Chaudhary, A. K., \& Ph, D. (2007). Bayesian Analysis of Two-Parameter Exponentiated Log-logistic Distribution, 25(1), 1-12. 
Cheng, R. C. H., \& Amin, N. A. K. (1979). Maximum product of spacings estimation with applications to the lognormal distribution. Math Report, 791.

Cheng, R. C. H., \& Amin, N. A. K. (1983). Estimating parameters in continuous univariate distributions with a shifted origin. Journal of the Royal Statistical Society: Series B (Methodological), 45(3), 394-403.

Choudhury, A. (2005). A simple derivation of moments of the exponentiated Weibull distribution. Metrika, 62(1), 1722.

Collatt, D. (2003). David Collett Modelling Survival Data in Medical Research - Copy.pdf.

Cordeiro, G. M., \& de Castro, M. (2011). A new family of generalized distributions. Journal of Statistical Computation and Simulation, 81(7), 883-898. https://doi.org/10.1080/00949650903530745

Cordeiro, G. M., Edwin, E. M., \& Ramires, T. G. (2015). A new generalized Weibull family of distributions: mathematical properties and applications. Journal of Statistical Distributions and Applications, 2(1). https://doi.org/10.1186/s40488-015-0036-6

Cordeiro, G. M., \& Nadarajah, S. (2011). Closed-form expressions for moments of a class of beta generalized distributions. Brazilian Journal of Probability and Statistics, 25(1), 14-33. https://doi.org/10.1214/09-BJPS109

Cordeiro, G. M., Ortega, E. M. M., \& Cunha, D. C. C. (2013). The Exponentiated Generalized Class of Distributions. Journal of Data Science, 11(1), 109-142. https://doi.org/10.6339/JDS.2013.11(1).1086

De Brito, C. R., Rêgo, L. C., De Oliveira, W. R., \& Gomes-Silva, F. (2019). Method for generating distributions and classes of probability distributions: The univariate case. Hacettepe Journal of Mathematics and Statistics, 48(3), 897-930. https://doi.org/10.15672/HJMS.2018.619

de Santana, T. V. F., Ortega, E. M. M., Cordeiro, G. M., \& Silva, G. O. (2012). \{T\}he $\{\mathrm{K}\}$ umaraswamy- $\{\mathrm{L}\}$ og- $\{\mathrm{L}\}$ ogistic $\{\mathrm{D}\}$ istribution. $\{J\}$ ournal of $\{$ S $\}$ tatistical $\{T\}$ heory and $\{$ A $\}$ pplications, 11(3), 265-291.

Dey, S., Kumar, D., Anis, M. Z., Nadarajah, S., \& Okorie, I. (2021). A Review of Transmuted Distributions. Journal of the Indian Society for Probability and Statistics. https://doi.org/10.1007/s41096-021-00096-0

Diekmann, A. (1992). The log-logistic distribution as a model for social diffusion processes. Journal of Scientific and Industrial Research, 51, 285-290.

Ekawati, D., Warsono, W., \& Kurniasari, D. (2015). On the Moments, Cumulants, and Characteristic Function of the Log-Logistic Distribution. IPTEK The Journal for Technology and Science, 25(3).

Eugene, N., Lee, C., \& Famoye, F. (2002). BETA-NORMAL DISTRIBUTION AND ITS APPLICATIONS. Communications in Statistics - Theory and Methods, 31(4), 497-512. https://doi.org/10.1081/STA-120003130

Fang, Y. (2011). Asymptotic equivalence between cross-validations and Akaike information criteria in mixed-effects models. Journal of Data Science, 9(1), 15-21.

Fisk, P. R. (1961). The graduation of income distributions. Econometrica: Journal of the Econometric Society, $171-185$.

Ghitany, M. E. (1998). On a recent generalization of gamma distribution. Communications in Statistics - Theory and Methods, 27(1), 223-233. https://doi.org/10.1080/03610929808832662

Gholam, H. H. (2013). Characterizations of exponentiated distributions. Pakistan Journal of Statistics and Operation Research, 9(1), 17-24. https://doi.org/10.18187/pjsor.v9i1.435

Gompertz, B. (1825). XXIV. On the nature of the function expressive of the law of human mortality, and on a new mode of determining the value of life contingencies. In a letter to Francis Baily, Esq. FRS \&c. Philosophical Transactions of the Royal Society of London, (115), 513-583.

Granzotto, D. C. T., Louzada, F., \& Balakrishnan, N. (2017). Cubic rank transmuted distributions: inferential issues and applications. Journal of Statistical Computation and Simulation, 87(14), 2760-2778. https://doi.org/10.1080/00949655.2017.1344239

Granzotto, D. C. T., \& Louzada, F. (2015). The Transmuted Log-Logistic Distribution: Modeling, Inference, and an Application to a Polled Tabapua Race Time up to First Calving Data. Communications in Statistics - Theory and Methods, 44(16), 3387-3402. https://doi.org/10.1080/03610926.2013.775307

Gui, W. (2013). Marshall-olkin extended log-logistic distribution and its application in minification processes. Applied Mathematical Sciences, 7(77-80), 3947-3961. https://doi.org/10.12988/ams.2013.35268

Gupta, R. C., \& Akman, H. O. (1995). On the reliability studies of a weighted inverse Gaussian model. Journal of 
Statistical Planning and Inference, 48(1), 69-83.

Gupta, R. C., Akman, O., \& Lvin, S. (1999). A study of log-logistic model in survival analysis. Biometrical Journal: Journal of Mathematical Methods in Biosciences, 41(4), 431-443.

Gupta, R. C., Gupta, P. L., \& Gupta, R. D. (1998). Modeling failure time data by Lehman alternatives. Communications in Statistics-Theory and Methods, 27(4), 887-904.

Gupta, R. D., \& Kundu, D. (1999). Theory \& methods: Generalized exponential distributions. Australian \& New Zealand Journal of Statistics, 41(2), 173-188.

Gupta, R. D., \& Kundu, D. (2001). Exponentiated exponential family: an alternative to gamma and Weibull distributions. Biometrical Journal: Journal of Mathematical Methods in Biosciences, 43(1), 117-130.

Gupta, R. D., \& Kundu, D. (2009). Introduction of shape/skewness parameter (s) in a probability distribution. J Probab Stat Sci, 7, 153-171.

Gupta, R. D., \& Kundu, D. (2002). Generalized exponential distributions: statistical inferences. Journal of Statistical Theory and Applications, 1(1), 101-118.

Jones, M. C. (2009). Kumaraswamy's distribution: A beta-type distribution with some tractability advantages. Statistical Methodology, 6(1), 70-81. https://doi.org/10.1016/j.stamet.2008.04.001

Jones, M. C. (2004). Families of distributions arising from distributions of order statistics. Test, 13(1), 1-43.

Kawanishi, T. (2020). Maximum likelihood and the maximum product of spacings from the viewpoint of the method of weighted residuals. Computational and Applied Mathematics, 39(3). https://doi.org/10.1007/s40314-020-01179-7

Khan, S. A., \& Khosa, S. K. (2015). Generalized log-logistic proportional hazard model with applications in survival analysis. Journal of Statistical Distributions and Applications, 3(1). https://doi.org/10.1186/s40488-016-0054-Z

Kleiber, C., \& Kotz, S. (2003). Statistical size distributions in economics and actuarial sciences (Vol. 470). John Wiley \& Sons.

Kumaraswamy, P. (1980). A generalized probability density function for double-bounded random processes. Journal of Hydrology, 46(1-2), 79-88. https://doi.org/10.1016/0022-1694(80)90036-0

Lee, C., Famoye, F., \& Alzaatreh, A. Y. (2013). Methods for generating families of univariate continuous distributions in the recent decades. Wiley Interdisciplinary Reviews: Computational Statistics, 5(3), 219-238. https://doi.org/10.1002/wics.1255

Lee, E. T., \& Wang, J. (2003). Statistical methods for survival data analysis (Vol. 476). John Wiley \& Sons.

Lehmann, E. L. (1953). The power of rank tests. The Annals of Mathematical Statistics, 23-43.

Lemonte, A. J. (2014). The beta log-logistic distribution. Brazilian Journal of Probability and Statistics, $28(3), 313-332$. https://doi.org/10.1214/12-BJPS209

Lemonte, A. J., Barreto-Souza, W., \& Cordeiro, G. M. (2013). The exponentiated Kumaraswamy distribution and its log-transform. Brazilian Journal of Probability and Statistics, 27(1), 31-53. https://doi.org/10.1214/11-BJPS149

Lemonte, A. J., \& Cordeiro, G. M. (2013). An extended Lomax distribution. Statistics, 47(4), 800-816. https://doi.org/10.1080/02331888.2011.568119

Li, R., \& Nadarajah, S. (2020). A review of Student's t distribution and its generalizations. Empirical Economics, 58(3), 1461-1490. https://doi.org/10.1007/s00181-018-1570-0

Lima, S. R., \& Cordeiro, G. M. (2017). The extended log-logistic distribution: Properties and application. Anais Da Academia Brasileira de Ciencias, 89(1), 3-17. https://doi.org/10.1590/0001-3765201720150579

Little, C. L., Adams, M. R., Anderson, W. A., \& Cole, M. B. (1994). Application of a log-logistic model to describe the survival of Yersinia enterocolitica at sub-optima $\mathrm{pH}$ and temperature. International Journal of Food Microbiology, 22(1), 63-71.

Louzada, F., \& Granzotto, D. C. T. (2016). The transmuted log-logistic regression model: a new model for time up to first calving of cows. Statistical Papers, 57(3), 623-640. https://doi.org/10.1007/s00362-015-0671-5

Mahdavi, A., \& Kundu, D. (2017). A new method for generating distributions with an application to exponential distribution. Communications in Statistics - Theory and Methods, 46(13), 6543-6557. https://doi.org/10.1080/03610926.2015.1130839

Malik, A. S., \& Ahmad, S. P. (2020). An Extension of Log-Logistic Distribution for Analyzing Survival Data. Pakistan 
Journal of Statistics and Operation Research, 16(4), 789-801. https://doi.org/10.18187/PJSOR.V16I4.2961

Marshall, A. W., \& Olkin, I. (1997). A new method for adding a parameter to a family of distributions with application to the exponential and Weibull families. Biometrika, 84(3), 641-652.

McDonald, J. B. (2008). Some generalized functions for the size distribution of income. In Modeling income distributions and Lorenz curves (pp. 37-55). Springer.

Mendoza, N. V. R., Ortega, E. M. M., \& Cordeiro, G. M. (2016). The exponentiated-log-logistic geometric distribution: Dual activation. Communications in Statistics - Theory and Methods, 45(13), 3838-3859. https://doi.org/10.1080/03610926.2014.909937

Merovci, F., Alizadeh, M., \& Hamedani, G. G. (2016). Another generalized transmuted family of distributions: Properties and applications. Austrian Journal of Statistics, 45(3), 71-93. https://doi.org/10.17713/ajs.v45i3.109

Midhu, N. N., Sankaran, P. G., \& Nair, N. U. (2013). A class of distributions with the linear mean residual quantile function and it's generalizations. Statistical Methodology, 15, 1-24.

Mudholkar, G. S., \& Hutson, A. D. (1996). The exponentiated Weibull family: some properties and a flood data application. Communications in Statistics--Theory and Methods, 25(12), 3059-3083.

Mudholkar, G. S., \& Srivastava, D. K. (1993). Exponentiated Weibull family for analyzing bathtub failure-rate data. IEEE Transactions on Reliability, 42(2), 299-302.

Mudholkar, G. S., Srivastava, D. K., \& Freimer, M. (1995). The exponentiated Weibull family: A reanalysis of the bus-motor-failure data. Technometrics, 37(4), 436-445.

Muthulakshmi, S. (2013). Double sampling plan for Truncated Life test based on Kumaraswamy-Log-Logistic Distribution. IOSR Journal of Mathematics, 7(4), 29-37. https://doi.org/10.9790/5728-0742937

Nadarajah, S., Cordeiro, G. M., \& Ortega, E. M. M. (2013). The exponentiated Weibull distribution: A survey. Statistical Papers, 54(3), 839-877. https://doi.org/10.1007/s00362-012-0466-x

Nadarajah, S., Cordeiro, G. M., \& Ortega, E. M. M. (2015a). The exponentiated G geometric family of distributions. Journal of Statistical Computation and Simulation, 85(8), 1634-1650. https://doi.org/10.1080/00949655.2014.885977

Nadarajah, S., Cordeiro, G. M., \& Ortega, E. M. M. (2015b). The zografos-balakrishnan-G family of distributions: Mathematical properties and applications. Communications in Statistics - Theory and Methods, 44(1), 186-215. https://doi.org/10.1080/03610926.2012.740127

Nadarajah, S., \& Kotz, S. (2006). The exponentiated type distributions. Acta Applicandae Mathematica, 92(2), 97-111.

Nandram, B. (1989). Discrimination between the complementary log-log and logistic model for ordinal data. Communications in Statistics-Theory and Methods, 18(6), 2155-2164.

Nasiru, S. (2018). A New Generalization of Transformed-Transformer Family of Distributions. Retrieved from http://ir.jkuat.ac.ke:8080/bitstream/handle/123456789/4674/MS400-0002-16\%28THESIS FINAL\%29.pdf?sequence=1\&isAllowed=y

Nasiru, S., Mwita, P. N., \& Ngesa, O. (2019). Alpha power transformed Fréchet distribution. Applied Mathematics and Information Sciences, 13(1), 129-141. https://doi.org/10.18576/AMIS/130117

Oluyede, B. O., Foya, S., Warahena-Liyanage, G., \& Huang, S. (2016). The log-logistic weibull distribution with applications to lifetime data. Austrian Journal of Statistics, 45(3), 43-69. https://doi.org/10.17713/ajs.v45i3.107

Pham, H., \& Lai, C. D. (2007). On recent generalizations of the Weibull distribution. IEEE Transactions on Reliability, 56(3), 454-458. https://doi.org/10.1109/TR.2007.903352

Prentice, R. L. (1976). A Generalization of the Probit and Logit Methods for Dose Response Curves. Biometrics, 32(4), 761-768. https://doi.org/10.2307/2529262

Prentice, R. L., \& Kalbfleisch, J. D. (1979). Hazard rate models with covariates. Biometrics, 25-39.

Rahman, M. M., Al-Zahrani, B., \& Shahbaz, M. Q. (2018). A general transmuted family of distributions. Pakistan Journal of Statistics and Operation Research, 14(2), 463-481. https://doi.org/10.18187/pjsor.v14i2.2334

Rahman, M. M., Al-Zahrani, B., \& Shahbaz, M. Q. (2019). Cubic transmuted Weibull distribution: properties and applications. Annals of Data Science, 6(1), 83-102.

Rahman, M. M., Al-Zahrani, B., \& Shahbaz, M. Q. (2020). Cubic transmuted pareto distribution. Annals of Data Science, 7(1), 91-108. 
Rahman, M. M., Al-Zahrani, B., Shahbaz, S. H., \& Shahbaz, M. Q. (2020). Transmuted probability distributions: A review. Pakistan Journal of Statistics and Operation Research, 16(1), 83-94. https://doi.org/10.18187/PJSOR.V16I1.3217

Ramos, M. W. A. (2013). The Zografos-Balakrishnan Log-Logistic Distribution. Properties and Application, Journal of Statistical Theory and Applications, 12(3), 244-255.

Ranneby, B. (1984). The maximum spacing method. An estimation method related to the maximum likelihood method. Scandinavian Journal of Statistics, 93-112.

Rao, G. S., Kantam, R. R. L., Rosaiah, K., \& Prasad, S. V. (2012). Reliability test plans for type-II exponentiated log-logistic distribution. Journal of Reliability and Statistical Studies, 5(1), 55-64.

Reath, J., Dong, J., \& Wang, M. (2018). Improved parameter estimation of the log-logistic distribution with applications. Computational Statistics, 33(1), 339-356. https://doi.org/10.1007/s00180-017-0738-y

Rosaiah, K., Kantam, R. R. L., \& Kumar, S. (2006). Reliability test plans for exponentiated log-logistic distribution. Stochastics and Quality Control, 21(2), 279-289.

Rowinski, P. M., Strupczewski, W. G., \& Singh, V. P. (2002). A note on the applicability of log-Gumbel and log-logistic probability distributions in hydrological analyses: I. Known pdf. Hydrological Sciences Journal, 47(1), $107-122$.

Samuel, A. F. (2019). On Transmuted Four Parameters Generalized Log-Logistic Distribution. International Journal of Statistical Distributions and Applications, 5(2), 32. https://doi.org/10.11648/j.ijsd.20190502.12

Shakhatreh, M. K. (2018). A new three-parameter extension of the log-logistic distribution with applications to survival data. Communications in Statistics - Theory and Methods, 47(21), 5205-5226. https://doi.org/10.1080/03610926.2017.1388399

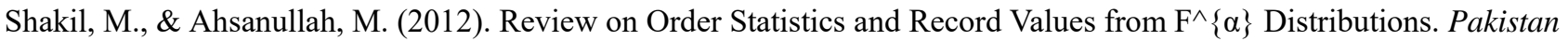
Journal of Statistics and Operation Research, 101-120.

Shaw, W. T., \& Buckley, I. R. C. (2009). The alchemy of probability distributions: beyond Gram-Charlier expansions, and a skew-kurtotic-normal distribution from a rank transmutation map, 1-8. Retrieved from http://arxiv.org/abs/0901.0434

Singh, K. P., Lee, C. M. -., \& George, E. O. (1988). On Generalized Log-Logistic Model for Censored Survival Data. Biometrical Journal, 30(7), 843-850. https://doi.org/10.1002/bimj.4710300714

Singh, U., Gupta, P. K., \& Upadhyay, S. K. (2005). Estimation of three-parameter exponentiated-Weibull distribution under type-II censoring. Journal of Statistical Planning and Inference, 134(2), 350-372.

Tahir, M.H., Mansoor, M., Zubair, M., \& Hamedani, G. G. (2014). McDonald log-logistic distribution with an application to breast cancer data. Journal of Statistical Theory and Applications, 13(1), 65. https://doi.org/10.2991/jsta.2014.13.1.6

Tahir, M. H., \& Cordeiro, G. M. (2015). Compounding of distributions: a survey and new generalized classes. Journal of Statistical Distributions and Applications (Vol. 3). Journal of Statistical Distributions and Applications. https://doi.org/10.1186/s40488-016-0052-1

Tahir, Muhammad H., \& Nadarajah, S. (2015). Parameter induction in continuous univariate distributions: Well-established G families. Anais Da Academia Brasileira de Ciencias, 87(2), 539-568. https://doi.org/10.1590/0001-3765201520140299

Teimouri, M. (2018). MPS: An R package for modelling new families of distributions. ArXiv Preprint ArXiv:1809.02959.

Thongkairat, S., Yamaka, W., \& Sriboonchitta, S. (2018). Maximum product spacings method for the estimation of parameters of linear regression. Journal of Physics: Conference Series, 1053(1). https://doi.org/10.1088/1742-6596/1053/1/012110

Tomy, L., Jose, M., \& Veena, G. (2020). A review on recent generalizations of exponential distribution, (October). https://doi.org/10.15406/bbij.2020.09.00313

Torabi, H., \& Hedesh, N. M. (2016). The gamma-uniform distribution and its applications Kybernetika Hamzeh Torabi ; Narges Montazeri Hedesh, (January 2012).

Verhulst, P.-F. (1838). Notice sur la loi que la population suit dans son accroissement. Corresp. Math. Phys., 10, 113126. 
Verhulst, P. (1847). Deuxième mémoire sur la loi d'accroissement de la population. Mémoires de l'académie Royale Des Sciences, Des Lettres et Des Beaux-Arts de Belgique, 20, 1-32.

Verhulst, P. F. (1845). Recherches Mathématiques sur La Loi D’Accroissement de la Population, Nouveaux Mémoires de l'Académie Royale des Sciences et Belles-Lettres de Bruxelles, 18, Art. 1, 1-45. N.

Zografos, K., \& Balakrishnan, N. (2009). On families of beta- and generalized gamma-generated distributions and associated inference. Statistical Methodology, 6(4), 344-362. https://doi.org/10.1016/j.stamet.2008.12.003

\section{Copyrights}

Copyright for this article is retained by the author(s), with first publication rights granted to the journal.

This is an open-access article distributed under the terms and conditions of the Creative Commons Attribution license (http://creativecommons.org/licenses/by/4.0/). 